\title{
A prognostics approach to nuclear component degradation modeling based on Gaussian Process Regression
}

\author{
Piero Baraldi, Francesca Mangili and Enrico Zio Member, IEEE
}

\begin{abstract}
Advanced diagnostics and prognostics tools are expected to play an important role in ensuring safe and long term operation in nuclear power plants. In this context, we use Gaussian Process Regression (GPR) to build a stochastic model of the equipment degradation evolution and apply it for prognostics.

GPR is a probabilistic technique for non-linear nonparametric regression that estimates the distribution of the future equipment degradation states by constraining a prior distribution to fit the available training data, based on Bayesian inference. Training data are taken from sequences of degradation measures collected from a set of similar historical equipments which have undergone a similar degradation process. Given new degradation measures from a currently degrading equipment (test trajectory), the distribution of the Remaining Useful Life (RUL) before failure is estimated by comparing with a failure criterion the distribution of the future degradation states predicted by GPR.

Applications are shown on simulated data concerning the evolution of creep damage in ferritic steel exposed to high stress and on real data concerning the clogging of sea water filters placed upstream the heat exchangers of a BWR condenser.
\end{abstract}

Index Terms - Remaining Useful Life, Prediction, Prognostics, Bayesian Inference, Gaussian Process Regression, Degradation, Ccreep, Ffilter Cclogging

\section{INTRODUCTION}

$\mathrm{N}^{\mathrm{u}}$ UCLEAR INDUSTRY is considering the development and use of advanced diagnostic and prognostic tools to enable longer term safe operation of nuclear structures, systems and components (SSCs) [1-7]. This interest is mainly originated by the following three reasons:

- many nuclear SSCs are becoming old due to the extension of the life of the existing reactors beyond the initial 30 or 40 years. This aging may cause degradation and even

P. Baraldi is with the Department of Energy, Politecnico di Milano, Italy (phone: +39 02 23996355; fax: +39 02 23996309; e-mail: piero.baraldi@polimi.it).

F. Mangili is with the Dalle Molle Institute for Artificial Intelligence (IDSIA), Scuola Universitaria Provessionale della Svizzera Italiana (SUPSI), Lugano, Switzerland, (e-mail: francesca@idsia.ch).

E. Zio is with the Department of Energy, Politecnico di Milano, Italy (email: enrico.zio@polimi.it) and the Chair on Systems Science and the Energetic challenge, European Foundation for New Energy-Electricite' de France, Ecole Centrale Paris and Supelec, France (e-mail: enrico.zio@ecp.fr). failures if SSC maintenance and replacement is not properly planned [8];

- many utilities are considering performing power up-rates in their plants. This typically requires increased coolant flow which may cause faster degradation of the SSCs;

- there is a strong economic interest towards having longer fuel cycles and decreased outage times. This may pose constraints on the frequency and extent of in-service inspections which may become not able to detect in a timely manner all SSC degradation modes.

In this context, in the present paper, we consider the development of prognostic methods for estimating the remaining useful life of nuclear components, structures and systems. Data-driven and model-based methods can be used for predicting the Remaining Useful Life (RUL) of degrading equipment [9-10], i.e., the remaining time during which the equipment can continue performing its function in a safe and efficient way. This allows the implementation of predictive maintenance strategies which have the potential of increasing safety and lowering costs [11]. Model-based methods assume that a mathematical model of the degradation process is available. In practice, the detailed knowledge necessary for building the model is available only for few, well-studied degradation mechanisms, and in fact, most often, equipment degrade for the interaction among different degradation mechanisms, which can be very hard to model [12]. Furthermore, these models have to explicitly account for the uncertainty in degradation evolution, e.g. arising due to scatter in microstructural properties and to variable loadings and external conditions [13].

On the other side, data-driven methods rely on the availability of observations collected during the degradation process of one or more similar pieces of equipment, from which the RUL prediction can be directly or indirectly derived. In the former case, Artificial Intelligence techniques, e.g., Artificial Neural Networks (ANNs) [14-15], similaritybased regression [16-17], etc., are used to directly map the relation between the observation and the equipment RUL. However, when the observations collected are directly related to the equipment degradation state, regression is used to extrapolate the future degradation path and compare it to a failure criterion. This approach provides an informative estimation of the entire degradation path, which can be checked against expert intuition to verify its consistency. 
Moreover, contrarily to direct RUL prediction, this approach can be applied even when no historical run- to- failure data are available.

Recent research works [18-19] show the effective use of ANNs also for degradation-based prognostic systems. A limit of ANNs models is that, in general, they do not provide an explicit quantification of the uncertainty of the predicted states, as do methods like Relevance Vector Machine (RVM) [20] or Gaussian Process Regression (GPR) [21-22]. Uncertainty is caused by model uncertainty (e.g., due to the limited amount of data used to build it), uncertainty on the observations (e.g., due to sensor noise), and process uncertainty (e.g., due to uncertain future loads and operating conditions) [13]. The intrinsic ability of RVM and GPR to fit probability distribution functions (pdfs) to the degradation data is desirable for prognostics where uncertainty management is of paramount importance [23-24]. In practice, the RVM method is actually a special case of a Gaussian Process (GP) [22].

The present paper proposes the use of GPR within a prognostic approach [25] that explicitly models the uncertainty in the future degradation states and provides the state prediction in the form of a Gaussian pdf. The hypothesis of Gaussianity is embraced to allow analytical calculations that make inference simpler. Although we have not always reasons to assume that a degradation process generates normally distributed degradation states, as long as there is no outstanding evidence to support the choice of a different state distribution, and GPR provides satisfactory predictions with reasonable uncertainty estimates, it might not be reasonable to make different assumptions that would only make the inference from data more complicate.

GPR can be used to predict the evolution in time of the distribution of the degradation state or of its rate of growth, which can be modeled as a function of time or of degradation. In this work, we propose a strategy to implement three approaches: 1) modeling the degradation state as a function of time; 2) modeling the degradation rate as a function of time; 3 ) modeling the degradation rate as a function of the degradation state. In any case, the final output is the prediction of the distribution of the future degradation states. By comparing the distribution obtained at different time instants during the degradation evolution with the value of a fixed failure threshold, i.e., the maximum value of degradation beyond which the equipment cannot be operated, it is possible to calculate the distribution of the equipment RUL.

The problem of inferring the degradation process of the test equipment from observations coming from pieces of equipment that are similar to it, but have followed degradation trajectories which are, in some extent, different, is faced by describing the degradation process as the composition of two elements: a general structure of the degradation trajectory, common to all pieces of equipment, and a variation around this structure which is different for each piece of equipment and thus uncorrelated between trajectories.

Two case studies are considered to validate the method and compare the three approaches proposed, in terms of the accuracy and precision of the RUL prediction and the reliability of the uncertainty estimate provided. In the first case study, the method is tested on simulated data generated by a non-linear model of creep growth in ferritic steel. In the second case study, we show the results of the application of GPR to real data concerning the clogging of filters used to clean the sea water pumped through the secondary side of a BWR condenser to cool the steam in the primary side.

The remaining of the paper is organized as follows: Section II describes the method for performing RUL predictions based on GPR and details the three approaches proposed for degradation modeling; Section III shows the results obtained by applying the GPR to the two case studies considered; finally, in Section IV we draw some conclusions and suggest potential future work.

\section{Methodology}

\section{A. Gaussian Process Regression}

GPR is a powerful and flexible approach to performing inference over functions $[22,26]$. In a regression problem, mapping from an input $x$ to an output $f(x)$, GPR defines the prior for the output $f(x)$ in the form of a distribution over functions specified by a Gaussian Process. A GP is a collection of random variables, any finite number of which has a joint Gaussian distribution. A real GP $f(x)$ is completely specified by its mean function $m(x)$ and covariance function $k\left(x, x^{\prime}\right)$ :

$$
\begin{aligned}
& f(\mathbf{x}) \sim \mathrm{GP}\{m(\mathbf{x}) ; K(\mathbf{x}, \mathbf{x})\} \\
& m(x)=E[f(x)] \\
& k\left(x, x^{\prime}\right)=E\left[(f(x)-m(x))\left(f\left(x^{\prime}\right)-m\left(x^{\prime}\right)\right)\right]
\end{aligned}
$$

where $\mathbf{x}$ represents a vector of input values and $K(\mathbf{x}, \mathbf{x})$ indicates the co-variance matrix containing the values of $k\left(x, x^{\prime}\right)$ evaluated for all possible pairs of inputs in $\mathbf{x}$.

This prior is taken to represent our prior beliefs over the kind of functions we expect to observe. Typically the prior mean and co-variance functions that we use will have some free parameters, called, usually, hyper-parameters. Although the choice of covariance function must be specified by the user, various methods have been proposed for determining the corresponding hyper-parameters from training data [22], e.g., the conjugate gradient optimizer that maximizes the marginal likelihood of the training set with respect to the hyperparameters.

Given the prior information about the GP, the value of the hyper-parameters and a set of training data $\mathbf{D}_{x y}=(\mathbf{x}, \mathbf{y})=$ $\left\{\left(x_{i}, y_{i}\right)\right\}_{i=1: N}$, the posterior distribution over functions is derived by imposing a restriction on the prior distribution to contain only those functions that agree with the observed data [22]. In other words, we impose the output in correspondence of the test input vector $\mathbf{x}^{\text {tst }}$ to be drawn from the same GP as the training data $\mathbf{D}_{x y}$, and thus we have: 


$$
\begin{aligned}
& {\left[\begin{array}{c}
\mathbf{y}=f(\mathbf{x}) \\
\mathbf{f}^{t s t}=f\left(\mathbf{x}^{t s t}\right)
\end{array}\right] \sim} \\
& \operatorname{GP}\left\{\left[\begin{array}{c}
m(\mathbf{x}) \\
m\left(\mathbf{x}^{t s t}\right)
\end{array}\right] ;\left[\begin{array}{cc}
K(\mathbf{x}, \mathbf{x}) & K\left(\mathbf{x}, \mathbf{x}^{t s t}\right) \\
K\left(\mathbf{x}^{t s t}, \mathbf{x}\right) & K\left(\mathbf{x}^{t s t}, \mathbf{x}^{t s t}\right)
\end{array}\right]\right\}
\end{aligned}
$$

The presence of a white Gaussian noise $v_{i}$ with variance $\sigma_{v}^{2}$ on the observations $y_{i}$ can be accounted for by adding the noise variance to the co-variance function:

$\mathbf{y}=f(\mathbf{x})+\mathbf{v} \sim \mathrm{GP}\left\{m(\mathbf{x}) ; K(\mathbf{x}, \mathbf{x})+\sigma_{v}^{2} \mathbf{I}\right\}$

where $\mathbf{I}$ is the identity matrix.

From eq. (2), the posterior distribution of the output $\mathbf{f}^{t s t} \mid D$ in correspondence of the input vector $\mathbf{x}^{\text {tst }}$ can be derived [22]:

$\mathbf{f}^{t s t} \mid \mathrm{D} \sim N\left(\overline{\mathbf{f}^{t s t}}, \operatorname{cov}\left(\mathbf{f}^{t s t}\right)\right)$

where

$$
\begin{aligned}
\overline{\mathbf{f}^{t s t}} & =m\left(\mathbf{x}^{t s t}\right)+K\left(\mathbf{x}, \mathbf{x}^{t s t}\right)\left[K(\mathbf{x}, \mathbf{x})+\sigma_{v}^{2} \mathbf{I}\right]^{-1}(\mathbf{y}-m(\mathbf{x})) \\
\operatorname{cov}\left(\mathbf{f}^{t s t}\right) & =K\left(\mathbf{x}^{t s t}, \mathbf{x}^{t s t}\right) \\
& -K\left(\mathbf{x}^{t s t}, \mathbf{x}\right)\left[K(\mathbf{x}, \mathbf{x})+\sigma_{v}^{2} \mathbf{I}\right]^{-1} K\left(\mathbf{x}, \mathbf{x}^{t s t}\right)
\end{aligned}
$$

\section{B. Prognostic model}

It is assumed that $R$ training trajectories are available from measurements collected during the process of degradation of $R$ pieces of equipment similar to the one of interest (test equipment). Each reference trajectory $r=1: R$ is made of a sequence $z_{1: n^{r}}^{r}$ of observations $z_{j}^{r}$ directly related to the degradation state $\delta_{j}^{r}$ of the $r$-th equipment at time $\tau_{j}$, $j=1: n^{r}$, where $\tau_{n^{r}}$ is the last measurement time before failure. Equipment fails when its degradation exceeds the failure threshold value $\delta^{\text {th }}$; let $\tau_{F}^{r}$ indicate the time at which the failure of the $r$-th piece of equipment occurs. A sequence of observations $z_{1: J}^{\text {test }}$ from $\tau_{1}$ to the present time $\tau_{J}$ is available also for the test equipment.

The goal of the GPR prognostic model is to predict the future degradation states of the equipment of interest and from them compute its RUL. In presence of uncertainties, e.g., due to the scatter in the microstructural and manufacturing characteristics, the loading and external conditions variability, etc., the damage state, at any time instant, is better represented by a random variable $\Delta(\tau)$ rather than by a deterministic quantity [25]. As a consequence, also the equipment RUL at the present time $\tau_{J}$ should be represented by a random variable $\operatorname{RUL}\left(\tau_{J}\right)$ [23].

In this work, we assume the distributions of the degradation states to be Gaussian with different mean $\bar{\Delta}(\tau)$ and variance $\sigma_{\Delta}^{2}(\tau)$ at every time instant $\tau$, and use the GPR method to evaluate the conditional probability density function (pdf) $p_{\Delta^{\text {test }}(t)}\left(\delta(t) \mid \mathbf{D}_{z}, z_{1: J}^{\text {test }}\right)$ of the future damage state $\Delta^{\text {test }}(\tau)$, $\tau>\tau_{p}$ given the training dataset $\mathbf{D}_{z}=\left\{z_{1: n^{r}}^{r}\right\}_{R=1: r}$, and the test trajectory $z_{1: J}^{\text {test }}$. Three different approaches are considered to infer the conditional pdf $p_{\Delta^{\text {test }}(t)}\left(\delta(t) \mid \mathbf{D}_{z}, z_{1: J}^{\text {test }}\right)$ from data, based on GPR:

1) We directly model the degradation state $\Delta(\tau)$ as a function of time by defining the prior:

$$
\Delta(\boldsymbol{\tau}) \sim \operatorname{GP}\left\{m_{\Delta}(\boldsymbol{\tau}) ; K_{\Delta}(\boldsymbol{\tau}, \boldsymbol{\tau})\right\}
$$

where $\tau$ is a vector of time instants. The hyperparameters of $m_{\Delta}(\boldsymbol{\tau})$ and $K_{\Delta}(\boldsymbol{\tau}, \boldsymbol{\tau})$ are optimized using the set of training data $\mathbf{D}_{\tau / z}\left\{\left(\tau_{j} ; z_{j}^{r}\right\}_{1: n^{r} ; r=1: R}\right.$ derived from the training trajectories. Finally, predictions of the mean $\bar{\Delta}(\tau)$ and the variance $\sigma_{\Delta}^{2}(\tau)$ of the pdf $p_{\Delta^{\text {test }}(t)}\left(\delta(t) \mid \mathbf{D}_{z}, z_{1: J}^{\text {test }}\right)$ of future degradation states are obtained from eq. (5) by conditioning the GP in eq. (6) on the training data in $\mathbf{D}_{\tau / z}$ and the data available from the part of test trajectory already observed: $\left\{\left(\tau_{j} ; z_{j}^{\text {test }}\right\}_{1: J}\right.$.

2) We model the degradation rate $d \Delta$ as a function of time by defining the prior:

$d \Delta(\boldsymbol{\tau}) \sim \operatorname{GP}\left\{m_{d \Delta}(\tau) ; K_{d \Delta}(\tau, \tau)\right\}$

The hyper-parameters of $m_{d \Delta}(\boldsymbol{\tau})$ and $K_{d \Delta}(\boldsymbol{\tau}, \boldsymbol{\tau})$ are optimized using the set of training data $\mathbf{D}_{\tau / d z}=$ $\left\{\left(\tau_{j} ;\left(z_{j+1}^{r}-z_{j}^{r}\right) /\left(\tau_{j+1}-\tau_{j}\right)\right)\right\}_{j=1: n^{r}-1 ; r=1: R}$ derived from the training trajectories. Prediction of the mean $\overline{d \Delta}\left(\tau^{\prime}\right)$ and the variance $\sigma_{d \Delta}^{2}\left(\tau^{\prime}\right)$ of the degradation rate pdf are obtained from eq. (5) by conditioning the GP in eq. (7) on the training data in $\mathbf{D}_{\tau / d z}$ and the data available from the part of test trajectory: $\left\{\left(\tau_{j} ;\left(z_{j+1}^{\text {test }}-z_{j}^{\text {test }}\right) /\left(\tau_{j+1}-\tau_{j}\right)\right)\right\}_{j=1: J-1}$. By integrating the mean $\overline{d \Delta}\left(\tau^{\prime}\right)$ and the variance $\sigma_{d \Delta}^{2}\left(\tau^{\prime}\right)$ of the degradation rate from the current time $\tau_{J}$ up to time $\tau$ it is possible to predict the mean $\bar{\Delta}(\tau)$ and the variance $\sigma_{\Delta}^{2}(\tau)$ of the pdf of interest $p_{\Delta^{\text {test }}(t)}\left(\delta(t) \mid \mathbf{D}_{z}, z_{1: J}^{\text {test }}\right)$ : 


$$
\begin{aligned}
& \bar{\Delta}(\tau)=z_{J}^{t e s t}+\int_{\tau_{p}}^{\tau} \overline{d \Delta}\left(\tau^{\prime}\right) \mathrm{d} \delta\left(\tau^{\prime}\right) \\
& \sigma_{\Delta}^{2}(\tau)=\int_{\tau_{p}}^{\tau} \sigma_{d \Delta}^{2}\left(\tau^{\prime}\right) \mathrm{d} \delta\left(\tau^{\prime}\right)
\end{aligned}
$$

In practice, time can be discretized so that the integrals in eq. (8) become summations over finite increments $\overline{d \Delta}\left(\tau^{\prime}{ }_{j}\right)\left(\tau^{\prime}{ }_{j+1}-\tau^{\prime}{ }_{j}\right)$.

3) We model the degradation rate $d \Delta$ as a function of the degradation state by defining the prior:

$d \Delta(\boldsymbol{\delta}) \sim \operatorname{GP}\left\{m_{d \Delta}(\boldsymbol{\delta}) ; K_{d \Delta}(\boldsymbol{\delta}, \boldsymbol{\delta})\right\}$

where $\boldsymbol{\delta}$ is a vector of degradation states. The hyperparameters of $m_{d \Delta}(\boldsymbol{\delta})$ and $K_{d \Delta}(\boldsymbol{\delta}, \boldsymbol{\delta})$ are optimized using the set of training data $\mathbf{D}_{z / d z}=$ $\left\{\left(z_{j}^{r} ;\left(z_{j+1}^{r}-z_{j}^{r}\right) /\left(\tau_{j+1}-\tau_{j}\right)\right)\right\}_{j=1: n^{r}-1 ; r=1: R}$ derived from the training trajectories. Predictions of the mean $\overline{d \Delta}(\delta)$ and the variance $\sigma_{d \Delta}^{2}(\delta)$ of the degradation rate pdf are obtained from eq. (5) for any value of degradation $\delta$ in input by conditioning the GP in eq. (9) on the training data in $\mathbf{D}_{z / d z}$ and on the test data $\left\{\left(z_{j}^{\text {test }} ;\left(z_{j+1}^{\text {test }}-z_{j}^{\text {test }}\right) /\left(\tau_{j+1}-\tau_{j}\right)\right)\right\}_{j=1: J-1}$. The mean $\bar{\Delta}(\tau)$ and the variance $\sigma_{\Delta}^{2}(\tau)$ of the pdf of interest $p_{\Delta^{\text {test }}(t)}\left(\delta(t) \mid \mathbf{D}_{z}, z_{1: J}^{\text {test }}\right)$ are obtained as the average and the variance of the degradation states of a large number $N^{\text {samp }}$ of degradation trajectories sampled from:

$$
\begin{gathered}
\boldsymbol{\delta}_{j+1}=\overline{d \Delta}\left(\delta_{j}\right)+v_{j} \\
v_{j} \sim N\left(0, \sigma_{d \Delta}^{2}\left(\delta_{j}\right)\right)
\end{gathered}
$$

starting from the last observed degradation state $z_{J}^{\text {test }}$ at time $\tau_{J}$ and using the Monte Carlo sampling algorithm.

Notice that, in this particular case where data are available both from historical (training) equipments and from the currently degrading (test) equipment, we aim to learn about the common structure underlying all degradation processes from the test trajectories available, but also to draw from the test trajectory the information about the specific variation around this structure that characterizes the equipment of interest.

To achieve this, the covariance function is built as follows [28]:

$$
\begin{aligned}
k\left(x_{j}^{r}, x_{j^{\prime}}^{r^{\prime}}\right)= & k_{1}\left(x_{j}^{r}, x_{j^{\prime}}^{r^{\prime}}\right)+k_{2}\left(x_{j}^{r}, x_{j^{\prime}}^{r^{\prime}}\right) \delta\left(r, r^{\prime}\right) \\
& +\sigma_{y}^{2} \delta\left(r, r^{\prime}\right) \delta\left({ }^{j},{ }^{\prime}{ }^{\prime}\right)
\end{aligned}
$$

where $x$ and $y$ are the input and output specific to each approach adopted and the reference index assigned to the test trajectory is $r=R+1$. The first term of the kernel corresponds to the covariance associated with the common structure underlying all degradation trajectories; the second represents the covariance owing to the variation of each trajectory around the common structure of a degradation process. This term assumes a finite value only when $x_{j}^{r}$ and $x_{j}^{r^{\prime}}$, are taken from the same trajectory, since we assume the variation specific to each trajectory to be uncorrelated across trajectories. Finally, the third term accounts for the noise associated with the observation of a specific output $y$.

The functions chosen to represent our prior on the mean and covariance of the GP are fundamental ingredients for the correct application of GPR. In the literature about GPR, the prior on the mean is often set to the constant value of zero, after appropriate normalization of the training data [22, 29]. However, this does not seem a convenient choice when the function to regress has an evident trend, as for degradation modeling. In these cases the prior mean function is chosen to accurately represent the trend (linear, power low, etc.) of the function to regress, which can be often guessed from a graphical representation of the training data. Notice, however, that imposing a particular functional form for $m(x)$ does not constrain the updated mean $\overline{\mathbf{f}^{t s t}}$ of the output variable to follow that same functional form.

The efficacy of different combinations of mean and covariance functions in modeling a set of training data can be evaluated based on the marginal likelihood obtained by each of them [22].

Given the value of the failure threshold, assumed here to be known, and the conditional distribution of the degradation state $p_{\Delta^{\text {test }}(t)}\left(\delta(t) \mid \mathbf{D}_{z}, z_{1: J}^{\text {test }}\right)$, the RUL cumulative distribution function (cdf) $P_{R U L\left(\tau_{J}\right)}\left(r u l_{J} \mid \mathbf{D}_{z}, z_{1: J}^{\text {test }}\right)$ is computed from $p_{\Delta(\tau)}\left(\delta(\tau) \mid \mathbf{D}_{z}, z_{1: J}^{\text {test }}\right)$ as the probability that the degradation $\Delta(\tau)$ at time $\tau=\tau_{J}+r u l_{J}$ exceeds the failure threshold $\delta^{t h}$ [27]:

$$
\begin{aligned}
P_{R U L\left(\tau_{J}\right)}\left(r u l_{J}\right. & \left.\mid \mathbf{D}_{z}, z_{1: J}^{\text {test }}\right) \\
& =\operatorname{Prob}\left(\operatorname{RUL}\left(\tau_{J}\right)<r u l_{J} \mid \mathbf{D}_{z}, z_{1: J}^{\text {test }}\right) \\
& =\int_{\delta^{\text {th }}}^{+\infty} p_{\Delta^{\text {test }}\left(t_{J}+r u l_{J}\right)}\left(\delta(\tau) \mid \mathbf{D}_{z}, z_{1: J}^{\text {test }}\right) \mathrm{d} \delta(\tau) \\
& =1-\Phi\left(\frac{\delta^{\text {th }}-\bar{\Delta}\left(t_{J}+r u l_{J}\right)}{\sigma_{\Delta}\left(t_{J}+r u l_{J}\right)}\right)
\end{aligned}
$$

where $\Phi$ is the standard normal cdf.

From the RUL cdf one can derive the prediction $r \hat{u} l_{J}$ of the equipment RUL as the mean value of the RUL distribution and the confidence interval $C I(\alpha)=\left[r u l_{j}^{\text {inf }}(\alpha), r u l_{j}^{\text {sup }}(\alpha)\right]$ containing the true value of the test equipment RUL, hereafter 
referred to as $r u l_{J}$, with probability $\alpha$.

\section{NUMERICAL APPLICATION}

In this Section, the three approaches proposed for using GPR within a prognostics framework are verified on data simulated using a non-linear model of creep growth in ferritic steel (Section III.A), and on real data taken from a case study about the clogging of filters in a BWR condenser (Section III.B).

The quality of the prediction depends from the choice of the mean and covariance functions. In this work, different choices for the mean and covariance functions have been compared based on their marginal likelihood. Notice that finding the optimal choice in absence of any knowledge about the actual process that governs the system can be a hard task. Since, to the best of our knowledge, a widely agreed procedure for the selection of GPR mean and covariance functions is not yet available in literature, we limited this choice to some reasonable combinations of covariance functions available from the literature [22] and picked out the one with the largest marginal likelihood, without guarantee that the choice is optimal and that the assumptions about the process are correct. The mean and covariance functions used in this work are listed in Appendix A.

Given $N^{t s t}$ test trajectories $\mathbf{z}_{1: J}^{q}, q=1: N^{t s t}$, and $N^{t r n}$ different sets of training trajectories $\mathbf{D}_{z}^{l}=\left\{\mathbf{z}_{1: n^{r}}^{r}, r=1: R\right\}$, $l=1: N^{t r n}$, each prognostic approach is used to provide the prediction $\quad r \hat{u} l_{J(\beta)}^{q} \mid \mathbf{D}_{z}^{l} \quad$ and $\quad C I_{J(\beta)}^{q}(\alpha) \mid \mathbf{D}_{z}^{l}, \quad$ in correspondence of the life fraction $\beta$, i.e., at time step $\tau_{J(\beta)}^{q}=\beta \cdot \tau_{F}^{q}$. Three performance indicators are evaluated by comparing such prediction with the true RUL value $r u l_{J(\beta)}^{q}$ :

1) The square root of the Mean Square Error (RMSE), i.e., the average value over all test trajectories $q=1: N^{t s t}$ and training sets $l=1: N^{\text {trn }}$ of the square error $\left(r \hat{u} l_{J(\beta)}^{q} \mid \mathbf{D}_{z}^{l}-r u l_{J(\beta)}^{q}\right)^{2}$ made in predicting the true RUL of the test equipment. The RMSE measures the accuracy of the prediction and is desired to be as small as possible.

2) The Coverage $\left(\operatorname{Cov}_{\alpha}\right)$ of the prediction interval $C I_{J(\beta)}^{q}(\alpha) \mid \mathbf{D}_{z}^{l}$, i.e., the percentage of times the condition $r u l_{J(\beta)}^{q} \in C I_{J(\beta)}^{q}(\alpha) \mid \mathbf{D}_{z}^{l} \quad$ is verified. This indicator measures the reliability of the confidence interval; we want the value of $\operatorname{Cov}_{\alpha}$ to be as close as possible to $\alpha$.

3) The amplitude $\left(M A_{\alpha}\right)$ of the confidence interval $C I_{J(\beta)}^{q}(\alpha) \mid \mathbf{D}_{z}^{l}$ averaged over all test trajectories $q=1: N^{t s t}$ and training sets $l=1: N^{t r n}$; this indicator gives a measure of the precision of the RUL prediction; in order to have a high precision, we wish to keep the value of $M A_{\alpha}$ as small as possible.

\section{A. Artificial dataset: creep growth in ferritic steel}

In this Section, GPR is applied to simulated degradation trajectories representing the accumulation of creep damage in ferritic steels, which are widely used for welded steam pipes in the construction of power plant components that operate under high temperatures and loads; in such conditions, the creep deformation and rupture are important factors in determining the equipment lifetimes.

\section{1) Creep growth models}

The evolution of creep damage in ferritic steel exposed to the load $\sigma$ is simulated using the uni-axial form of the nonlinear creep constitutive equations proposed within the framework of Continuum Damage Mechanics by Mustata \& Hayhurst [30]:

$$
\left\{\begin{array}{l}
\dot{\varepsilon}=A \sinh \left[\frac{B \sigma(1-H)}{(1-\phi)(1-\omega)}\right] \\
\dot{H}=\frac{h \dot{\varepsilon}}{\sigma}\left(1-\frac{H}{H^{*}}\right) \\
\dot{\phi}=\frac{K_{c}}{3}(1-\phi)^{4} \\
\dot{\omega}=C \dot{\varepsilon}
\end{array}\right.
$$

where $\varepsilon$ is the creep strain, i.e., the fraction of elongation of the metallic piece in the longitudinal direction with respect to its original length, $\phi$ and $\omega$ are two damage state variables describing, respectively, the coarsening of the carbide precipitates, and the inter-granular creep constrained cavitation damage, $H$ is the hardening state variable, used to represent the strain hardening effect attributed to primary creep, and $A, B, H^{*}, h, K_{c}$ and $C$ are material inherent characteristics. Each characteristic $\varphi_{1: 6}=A, B, H^{*}, h, K_{c}, C$ varies with the temperature according to the Arrhenius law, i.e., $\varphi_{m}=\varphi_{m 0} \exp \left(-Q_{m} / T\right), \quad m=1: 6$, where $T$ is the operating temperature and $\varphi_{m 0}$ and $Q_{m}$ are parameters whose values have to be determined experimentally.

To generate different trajectories, the intrinsic variability of the creep growth process is simulated by sampling the values of the load $\sigma$ and temperature $T$ to which the steel is exposed at each time step from a normal distribution centered on their mean value, whereas the variability of the creep growth process between similar pieces of equipment is simulated by sampling the value of parameters $\varphi_{m 0}$ and $Q_{m}$, $m=1: 6$, at the beginning of each new simulated trajectory. We assume failure to happen when the limiting creep strain value of 0.02 is reached. Finally, to generate the sequence of observations $\quad \mathbf{z}_{1: n^{r}}^{r}=\left\{\varepsilon\left(\tau_{j}\right)+v_{j}\right\}_{j=1: n^{r}}^{r}$, collected one every 100 days, a white Gaussian noise $v_{j}$ with standard deviation $\sigma_{v}=2 \times 10^{-4}$ is added to the simulated creep strain $\varepsilon\left(\tau_{j}\right)$ at the observation time $\tau_{j}$. The time interval between two 
observations is rather large; in practical applications, this can happen when the costs of an inspection are elevated. Here this choice was done to limit the number of training data, since computational costs of GPR scale typically as $O\left(N^{3}\right)$ with the number $N$ of training data. Fig. 1 shows an example of simulated creep growth trajectory (top) and the corresponding sequence of observations $\mathbf{z}_{1: n^{r}}^{r}$ (bottom).

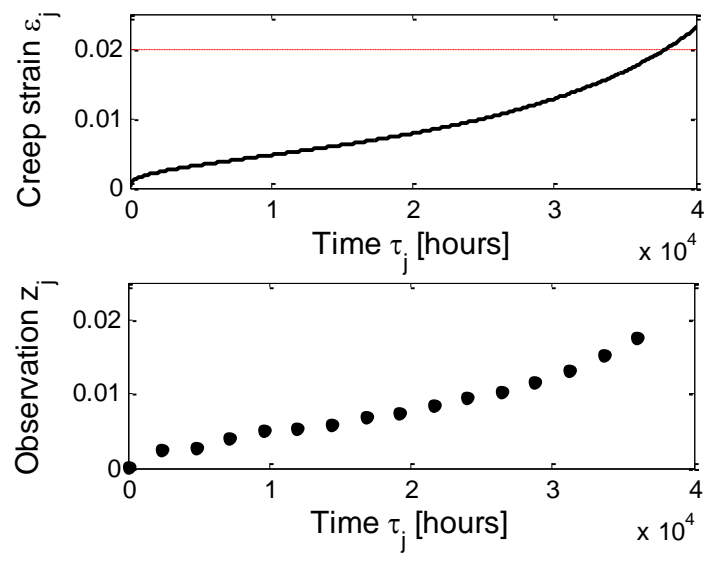

Fig. 1. Example of simulated creep growth trajectory (top) with the corresponding sequence of observations (bottom).

For a better evaluation of the method, we have adopted two different sets of parameters (see Appendix B for their distribution) to simulate trajectories with smaller or larger variability. Fig. 2 compares 10 trajectories with low (top) and high (bottom) variability.

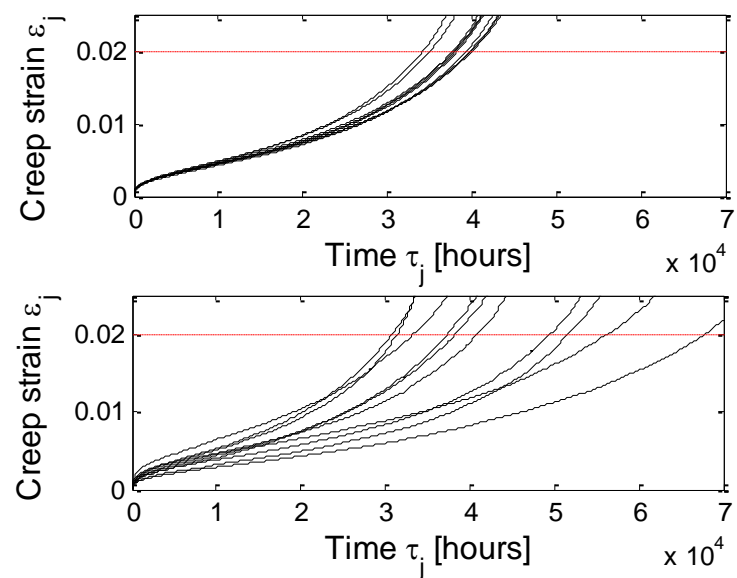

Fig. 2. Examples of creep growth trajectories with low (top) and high (bottom) variability.

\section{2) Results}

In this Section we show, first, some results obtained by applying the three approaches proposed in Section II.B to a single creep growth trajectory; for this, a set of $R=10$ training trajectories is used. In this first part, we consider only trajectories with low variability (Fig. 2, top). Then, we compare the performances obtained by the three approaches in correspondence of trajectories with low and high variability.
In Fig. 3, the predicted future evolution of degradation obtained by the GPR in approach 1 is compared with the true evolution of the test trajectory at two prediction time instants, $\tau_{1}=1$ hour (top) and $\tau_{12}=26400$ hours (bottom). Notice that the prediction accuracy is lower, i.e., the confidence interval is larger, for a prediction done at time $\tau_{1}=1$ since no data are available from the test trajectory, and the GPR can only account for the common structure of the degradation process drawn from the training trajectories; on the other side, at time $\tau_{12}=26400$ the precision increases, since GPR is able to learn its peculiar behavior from the data collected during the test trajectory thanks to the second term $k_{2}\left(x_{j}^{r}, x_{j^{\prime}}^{r^{\prime}}\right) \delta\left(r, r^{\prime}\right)$ of the covariance function in eq. (11). See Section III.A.3 for a further discussion of this aspect.
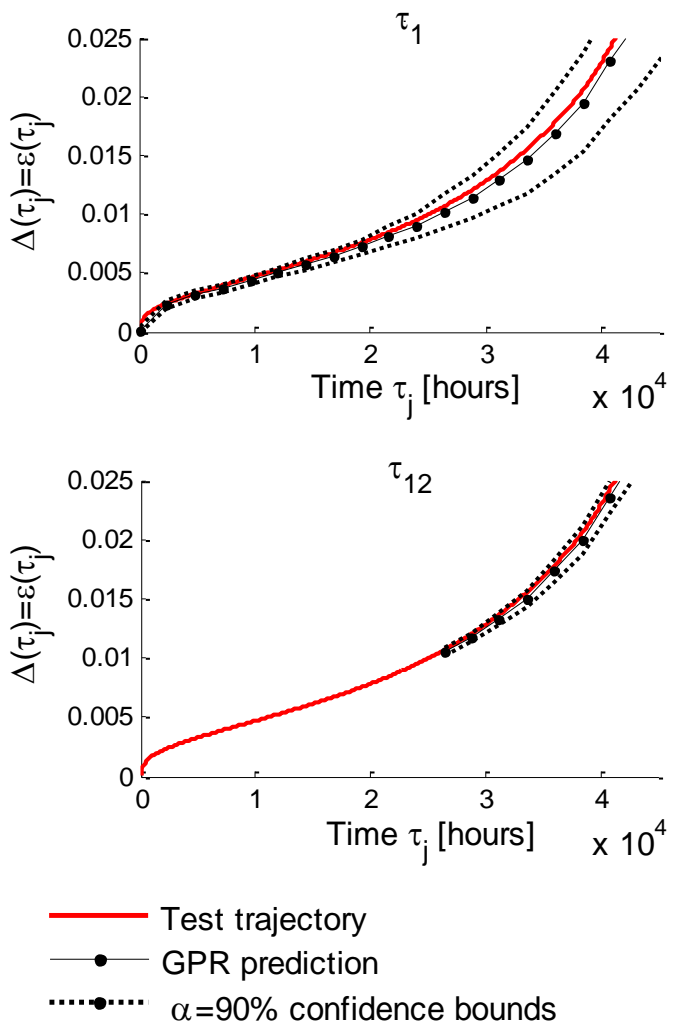

Fig. 3. GPR prediction of future degradation states obtained at two time instants $\tau_{1}=1$ hours (upper) and $\tau_{12}=26400$ hours (bottom) using approach 1 .

In approaches 2 and 3 , GPR is used to predict the degradation rate $d \Delta$ as a function of the time and the degradation state $\Delta$, respectively. In Figs. 4 and 5 , the predicted degradation rate (top) and the corresponding prediction of the future degradation evolution (bottom) are shown at the two time instants of Fig. 3 for approach 2; in Figs. 6 and 7 the same results are shown for approach 3. The predicted value of the degradation rate (continuous line) is compared with its true value (asterisks) observed for the test trajectory. 

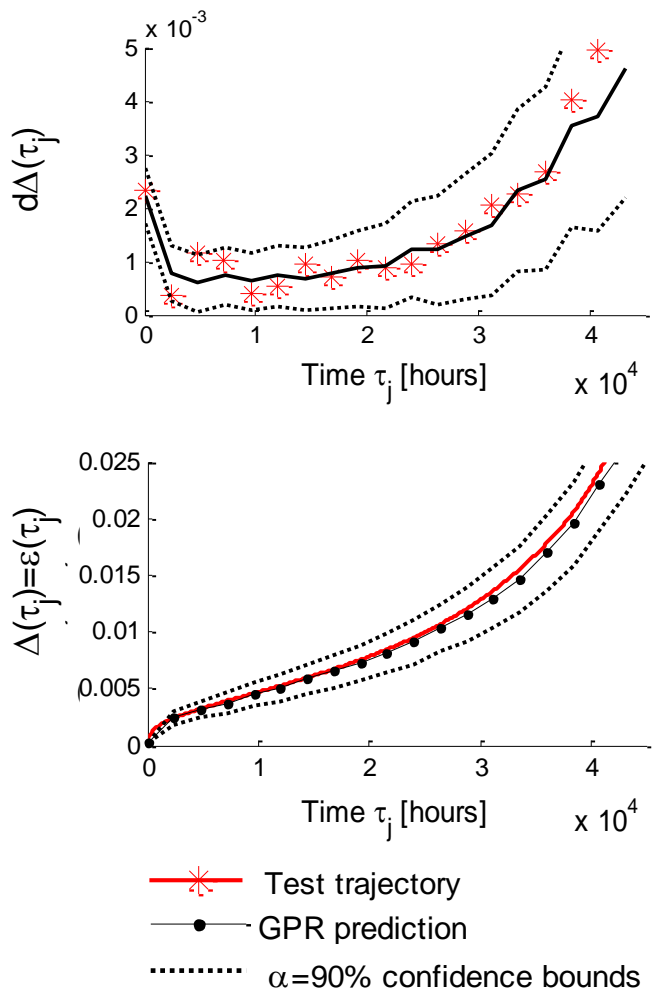

Fig. 4. GPR prediction of the degradation rate (top) and of the future degradation states (bottom) obtained at time instant $\tau_{1}=1$ hours using approach 2 .
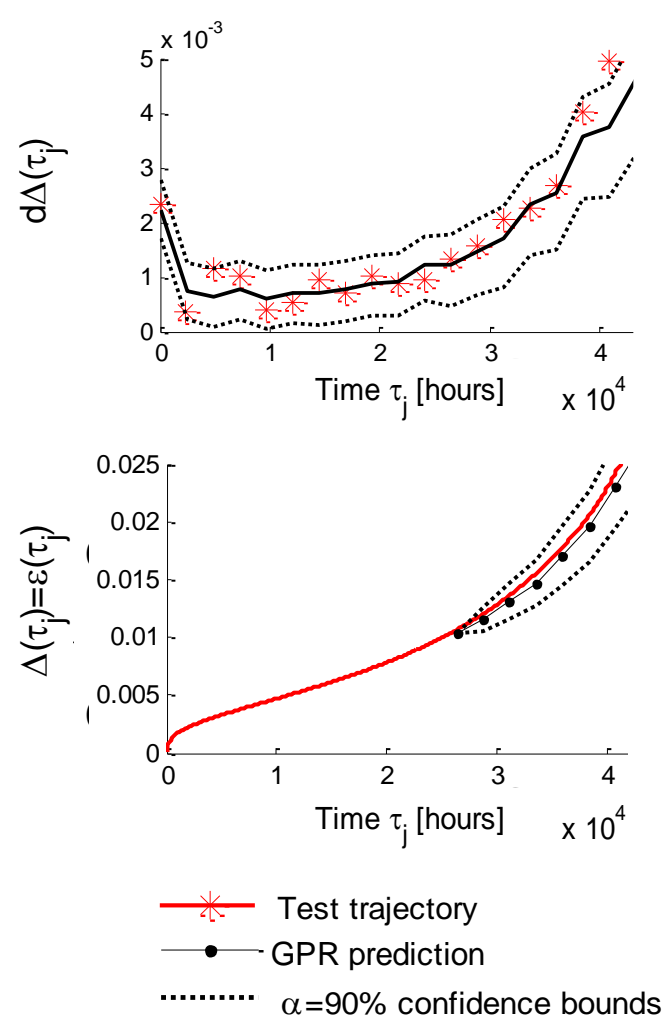

Fig. 5 GPR prediction of the degradation rate (top) and of the future degradation states (bottom) obtained at time instant $\tau_{12}=26400$ hours using approach 2 .
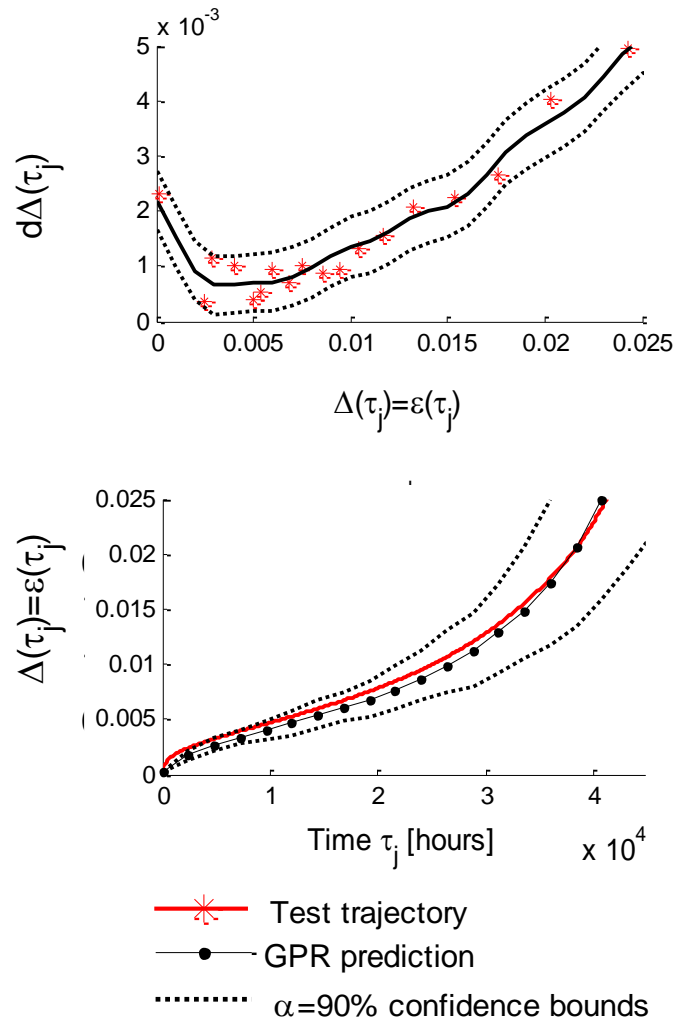

Fig. 6 GPR prediction of the degradation rate (top) and of the future degradation states (bottom) obtained at time instant $\tau_{1}=1$ hours using approach 3 .
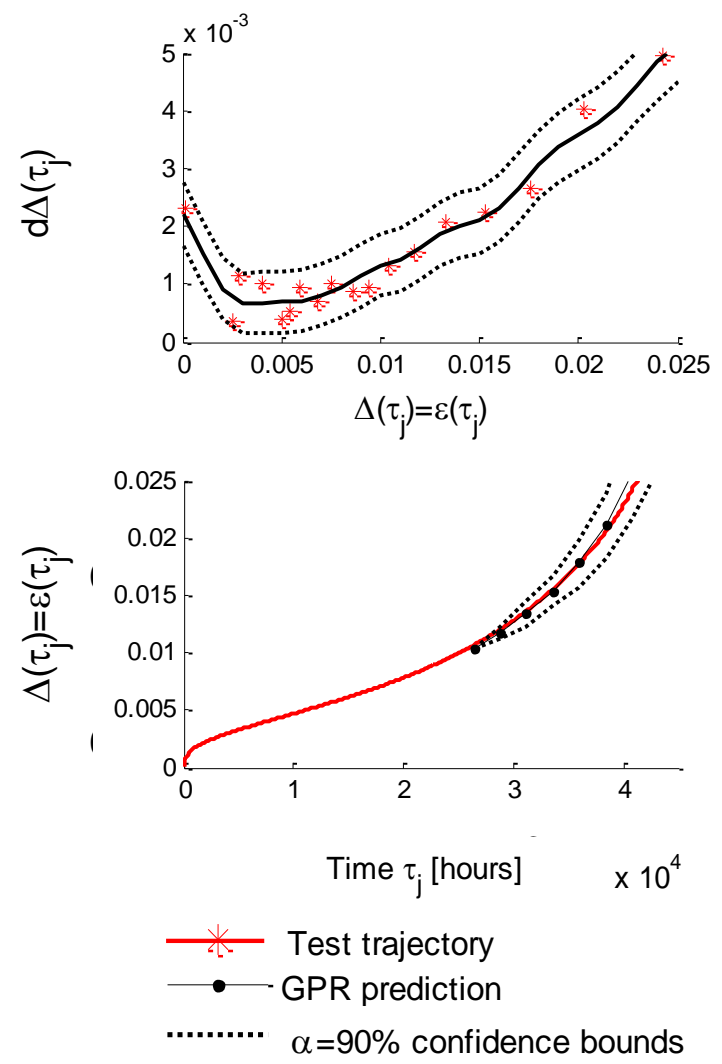

Fig. 7 GPR prediction of the degradation rate (top) and of the future degradation states (bottom) obtained at time instant $\tau_{12}=26400$ hours using approach 3 . 
Results show that these two approaches are less effective in learning the peculiar behavior of the test trajectory as more data are collected during its evolution. This could be due to the fact that the observed degradation rate is affected by a larger noise than the degradation state, since the relative effect of measurement and process noises is more evident on the small values of the degradation rates than on those, larger, of the degradation states. For this reason, after a sufficient amount of observations is collected, we expect approach 1 to provide narrower confidence intervals $C I(\alpha)$ than approaches 2 and 3 .

Fig. 8 compares the RUL prediction obtained by the three approaches at different time instants, with the true RUL value of the test trajectory considered. All three approaches can predict the equipment RUL fairly well, although the prediction of approach 3 appears less accurate in the first part of the trajectory. Approach 2 supplies narrower confidence intervals for the RUL prediction than the other two approaches at the beginning of the trajectory; however, the reliability of such interval has to be verified by checking that their coverage is close enough to the target value of $\alpha=0.9$.

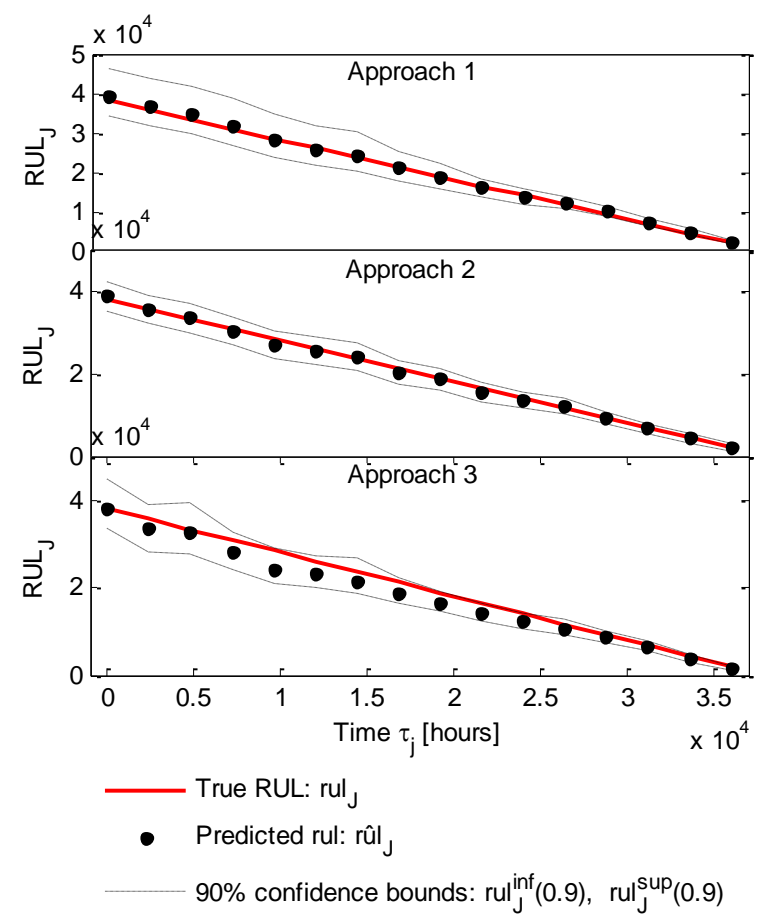

Fig. 8 Comparison at different time instants of the RUL prediction with the true RUL value for the three GPR-based approaches.

For a more robust evaluation of the performance of the three approaches proposed, the model in eq. (13) is used to generate $N^{t s t}=100$ test trajectories with low variability (Fig. 2 , top) and $N^{\text {trn }}=10$ different sets of $R=10$ similar training trajectories. For each training dataset simulated, the three approaches are applied to each test trajectory at four different life fractions $\beta_{1}=0.3, \beta_{2}=0.6, \beta_{3}=0.8$ and $\beta_{4}=0.95$, and the three performance indicators, $\operatorname{Cov}_{0.9}$, RMSE, and
$M A_{0.9}$ are computed. The same procedure is repeated simulating trajectories with high variability (Fig. 2, bottom). Figs. 9 and 10 show the values of the three performance indicators in case of low and high variability of the trajectories, respectively. The average value $m R U L$ of the RUL of the test trajectories at each life fraction $\beta$ is also shown for comparison with the values of the indicators RMSE and $M A_{0.9}$. A horizontal line indicates the target value $\alpha=0.9$ for the coverage (first from the top).
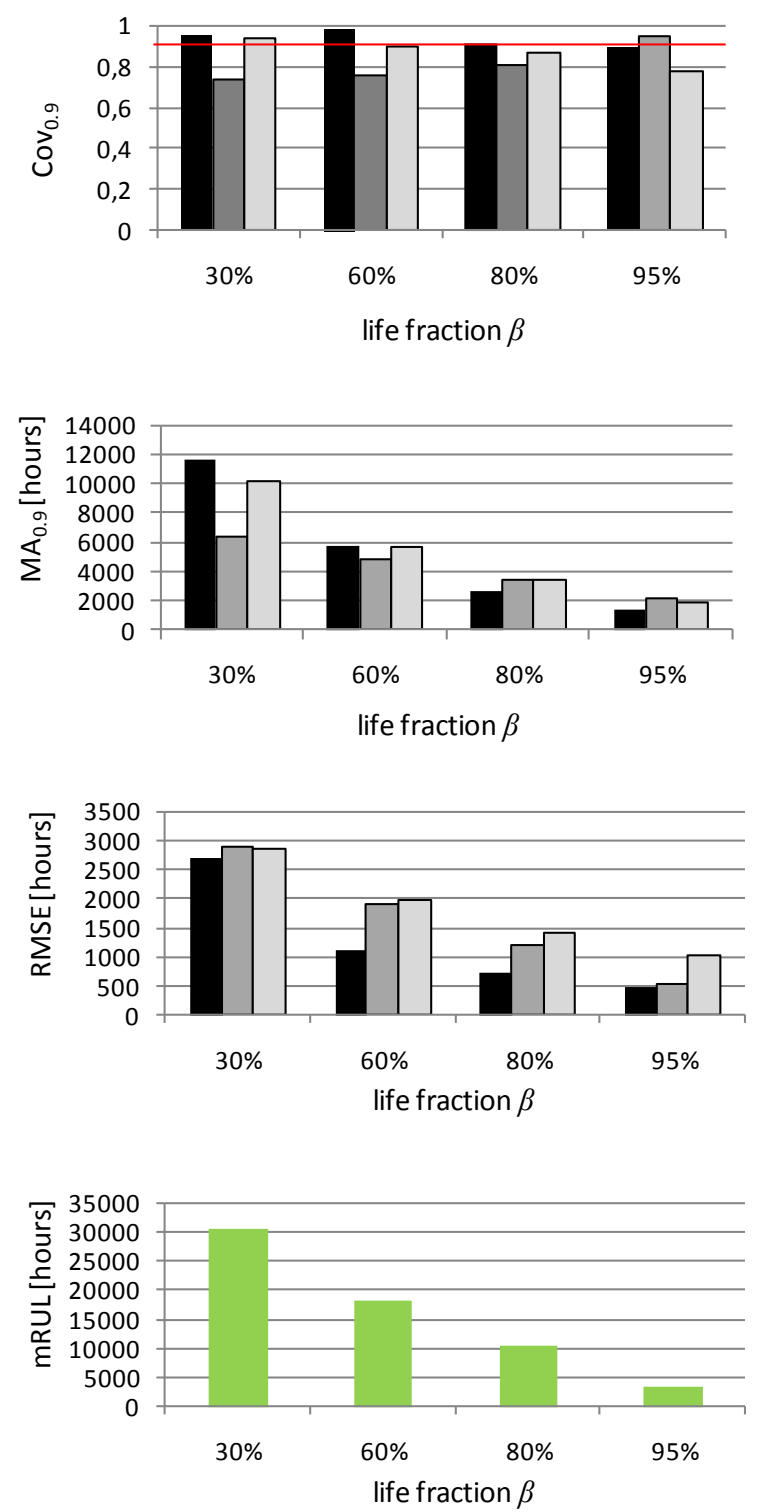

- Approach $1 \square$ Approach $2 \square$ Approach $3 \square$ Average RUL

Fig. 9 Comparison of the performance of the three approaches for trajectories with low variability. The horizontal line in the $\operatorname{Cov}_{0.9}$ figure (first from the top) indicates the target coverage value $\alpha=0.9$. 

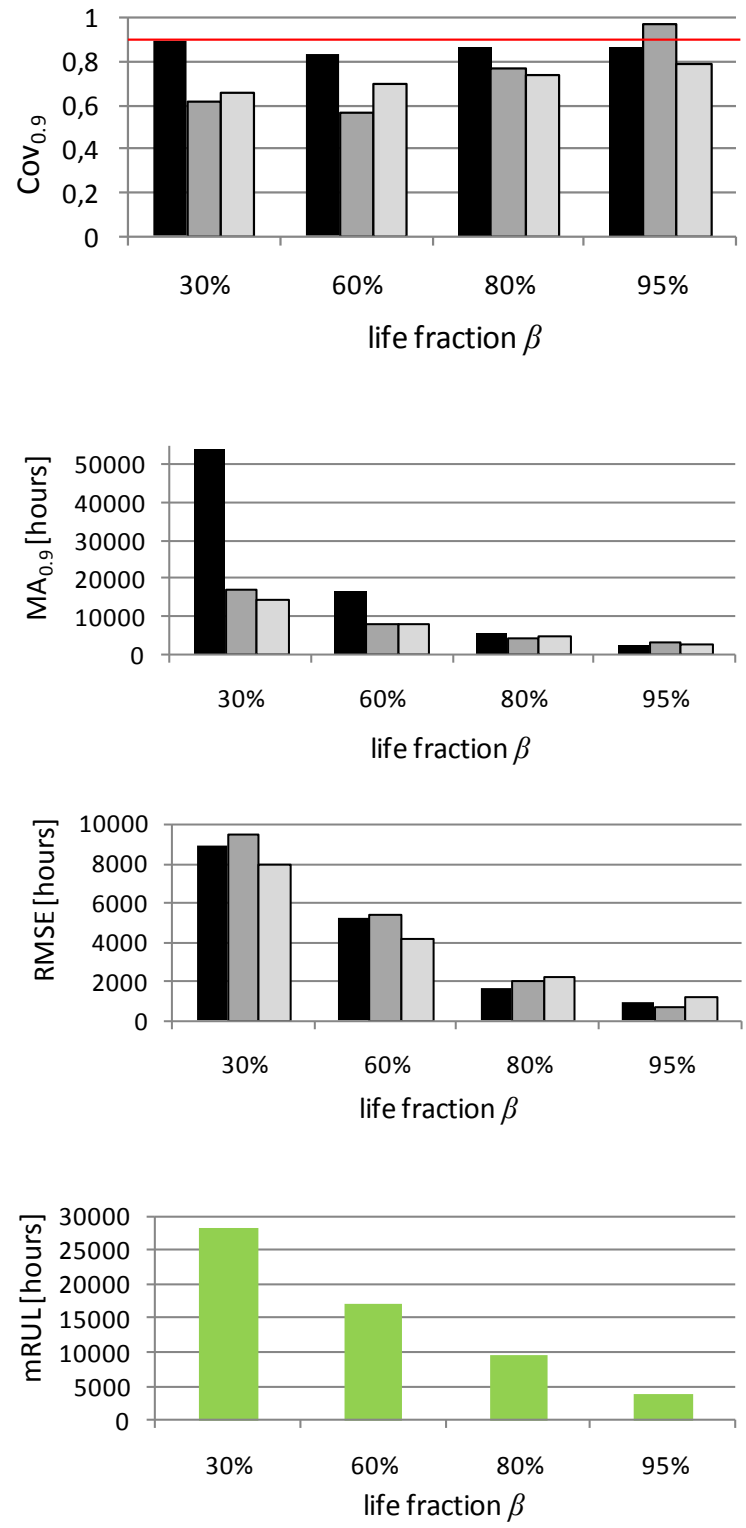

- Approach $1 \square$ Approach $2 \square$ Approach 3 $\square$ Average RUL

Fig. 10 Comparison of the performance of the three approaches for trajectories with high variability. The horizontal line in the $\operatorname{Cov}_{0.9}$ figure (first from the top) indicates the target coverage value $\alpha=0.9$.

In case of creep growth trajectories with low variability (Fig. 9), the error RMSE and the amplitude of the confidence interval $M A_{0.9}$ are always much smaller than the mean RUL, being around $10 \%$ and $30 \%$ of $m R U L$, respectively. As expected, the value of both indicators decreases with the life fraction $\beta$, since more data are available and failure is closer, and thus the effects of the process uncertainty on the accuracy and precision of the prediction are reduced. The increase in the accuracy and precision with the life fraction is more evident for approach 1 . This approach is able to provide always the most accurate predictions, and, towards the end-of-life of the equipment, even the narrowest confidence interval compared to the other two approaches. Moreover, it is also the only approach obtaining a coverage always close to $\alpha=0.9$. Approach 2 provides narrower confidence intervals at the beginning of the equipment life, but fails attaining the coverage value of 0.9 , so that we cannot rely on such narrow intervals. Finally, approach 3 obtains good coverage except for $\beta_{4}=0.95$, i.e., very close to the equipment failure, but is less accurate (larger RMSE) than approach 1.

Similar results are obtained when trajectories with large variability are considered (Fig. 10). Although in this case no approach is able to assure a coverage of 0.9 , approach 1 goes very close to this target, whereas approach 2 reaches such coverage only when the equipment is close to failure. The confidence interval supplied by approach 1 is rather large in the first part of the trajectory, with a value that, at $\beta=0.3$, is almost twice the average RUL value, mRUL. However, due to the large variability of the trajectories, such a large confidence interval may be necessary to correctly represent the intrinsic uncertainty of the future creep evolution. Finally, although in this case we cannot state that approach 1 is the most accurate and precise (at $\beta_{1}=0.3, \beta_{2}=0.6$ it is outperformed in accuracy by approach 3 , and by approach 2 at $\beta_{4}=0.95$ ), the RMSE is always comparable with that obtained by the other two approaches and does not exceed $30 \%$ of the average RUL, mRUL .

In this case study, all three approaches have shown the capability of tackling the prognostic problem and supplying accurate RUL prediction with associated measure of uncertainty. Approach 1 has outperformed the other two in accuracy. However, the fact that the coverage is often lower than its target value shows that the prediction uncertainty is not correctly quantified. Further research is necessary to identify how to correctly account for all sources of uncertainty affecting the RUL prediction; we suggest, in particular, that model uncertainty could play a significant role, especially in case of high variable trajectories. In this case, resorting to an ensemble of GPR models [29], could allow achieving higher reliability and improved prediction performance.

Notice finally that, as already mentioned, the choice of the covariance also impacts the performance of the method; the development of a procedure for the selection of GPR covariance functions is a fundamental requirement for the successful exploitation of GPR in prognostics.

\section{3) Analysis of the covariance function}

In this Section, we study the effects of the two terms $k_{1}\left(x_{j}^{r}, x_{j^{\prime}}^{\prime r^{\prime}}\right)$ and $k_{2}\left(x_{j}^{r}, x_{j^{\prime}}^{\prime r^{\prime}}\right) \delta\left(r, r^{\prime}\right)$ of the covariance function in eq. (11). For this, approach 1 has been applied using the covariance function in eq. (11) without the first term $k_{1}$, first, and then without the second term $k_{2}$. The predictions about the future degradation states provided by approach 1 without the first term $k_{1}$ or without the second term $k_{2}$ of the covariance function are shown in Figs. 11 and 12 , respectively, at time instant $\tau_{1}=1$ hours (upper) and $\tau_{12}=26400$ hours (bottom). 

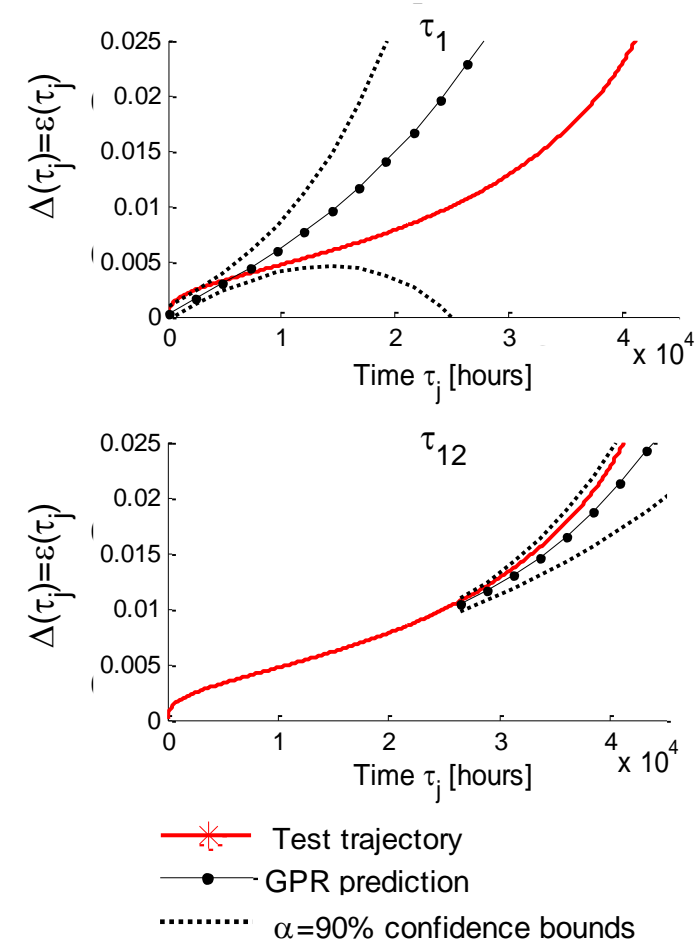

Fig. 11 GPR prediction of future degradation states obtained at two time instants $\tau_{1}=1$ hours (upper) and $\tau_{12}=26400$ hours (bottom) using approach 1 without the first term $k_{1}$ of the covariance function in eq. (11).
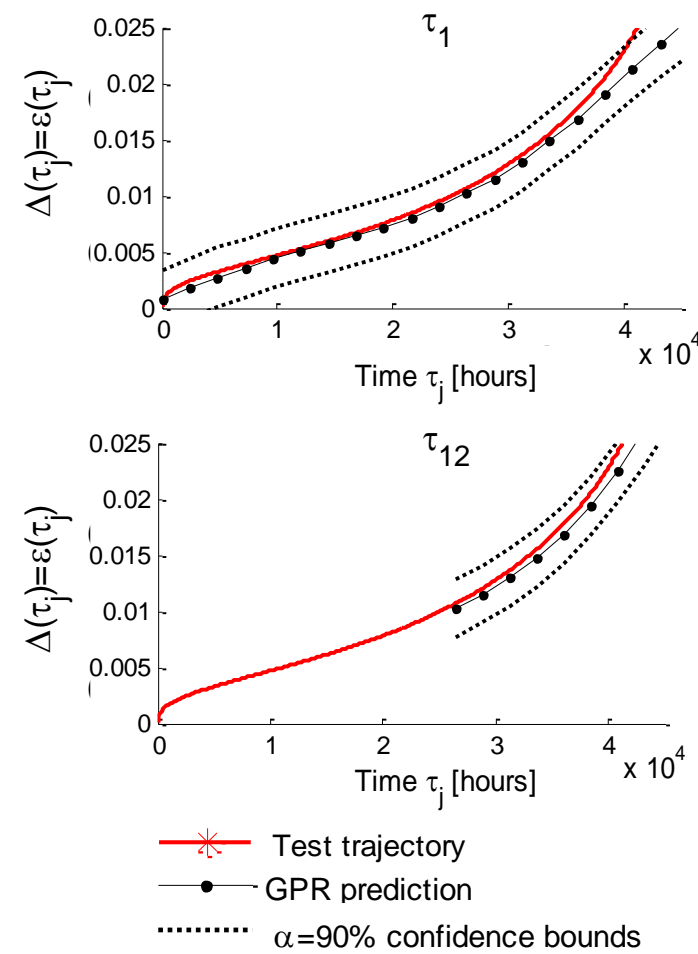

Fig. 12 GPR prediction of future degradation states obtained at two time instants $\tau_{1}=1$ hours (upper) and $\tau_{12}=26400$ hours (bottom) using approach 1 without the second term $k_{2}$ of the covariance function in eq (11).
These results show that if the first term $k_{1}$ of eq. (11), which refers to the common structure underlying all creep growth trajectories, is not considered (Fig. 11), the GPR model predictions at time $\tau_{1}=1$ hours are very uncertain (Fig. 11, top). This is due to the fact that no information is available about the test degradation trajectory, and at the same time the information coming from the training trajectory is not used for conditioning the prior GP distribution, but only for optimizing the hyper-parameters of the covariance function $k$. As more data about the test trajectory become available the prediction improves (Fig. 11, bottom), but remains, however, less accurate and precise than that in Fig. 3 (bottom), where the complete covariance function is used.

On the other hand, if we do not consider the second term $k_{2}$ of eq. (11) (Fig. 12), which refers to the variation of each trajectory around the common structure of the creep growth process, the GPR model predictions at time $\tau_{1}=1$ hours are less uncertain (Fig. 12, upper), but no improvement can be observed as more data become available (Fig. 12, bottom). In other words, the model is not able to learn the peculiar behavior of the test trajectory, since all observations, no matter which equipment they refer to, are given the same relevance when used for conditioning the prior GP distribution.

It is clear that the two terms $k_{1}$ and $k_{2}$ are complementary, and for this reason their combination assures better prediction performance along the entire duration of the degradation trajectory, as shown in Fig. 3.

\section{B. Real dataset: Clogging of BWR condenser filters}

In this Section, we consider the problem of predicting the RUL of filters used to clean the sea water entering the condenser of the BWR reactor of a Swedish nuclear power plant. During operations, filters undergo clogging and, once clogged, can cumulate particles, seaweeds, and mussels from the cooling water in the heat exchanger. For this reason, prompt and effective cleaning of the filter is desirable; predictive maintenance can help achieving this result, keeping maintenance costs reasonably low.

An increasing number of articles can be found in the literature concerning the study of filter clogging by solid aerosols [31] and liquid aerosols [32]. Common for these articles is that the results are achieved in a controlled environment: in the experimental setup all degradation quantities, indicators of degradation, and stressors are automatically measured and recorded. This is not the case in this industrial case study where, for a filter $q$ at time $\tau_{j}$, we only have available the measurements of the pressure drop $\Delta P_{j}^{q}$, and the flow across the filter $\dot{M}_{j}^{q}$. However, it has been well established that the clogging of a filter medium leads to an increase in pressure drop over the filter as long as the filtration velocity, and thus the flow, is kept constant. It is also known that the pressure drop is proportional to the square of the filtration velocity, and thus we take as an indicator of the state of clogging of filter $q$ at time $\tau_{j}$ the ratio [33]: 
$z_{j}^{q}=\frac{\Delta P_{j}^{q}}{\left(\dot{M}_{j}^{q}\right)^{2}}$

Fig. 13 shows the sequences of observations $\mathbf{z}_{1: n^{q}}^{q}$, $q=1: N^{t s t}$ collected on field during the clogging process of $N^{t s t}=8$ filters. We can see from this Figure that the clogging process is affected by large uncertainties, which can be ascribed to the very variable conditions of the sea water; in this context, the challenge is to provide sufficiently narrow confidence intervals for the values of the filters RUL.

Due to the absence of physical knowledge about the failure threshold, its value has been arbitrarily set to $\delta^{\text {th }}=175$ $\operatorname{bar} /\left(\mathrm{m}^{3} / \mathrm{s}\right)^{2}$.

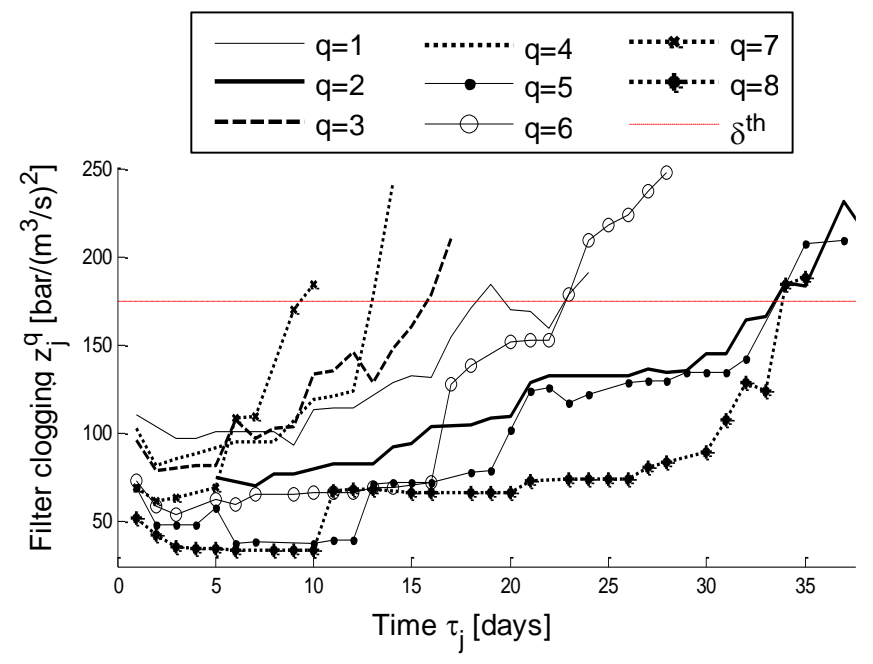

Fig. 13 available clogging trajectories $\mathbf{z}_{1: n^{q}}^{q}, q=1: 8$.

The three prognostic approaches previously presented are in turn applied at each time instant of each trajectory $q=1: 8$, using the remaining $R=7$ trajectories for training. Figs. 14, 15 and 16 show the RUL prediction obtained by approach 1, 2 and 3, respectively.

Due to the large uncertainty of the process, the accuracy of the RUL prediction is always rather low and the confidence intervals are very large in all three approaches. At a first glance, the outcomes of approaches 1 and 2 appear quite similar, except for trajectories $q=5$ and $q=8$ where approach 1 provides more accurate RUL predictions and narrower confidence intervals. In Fig. 16 the RUL predictions provided by Approach 3 appear, instead, more accurate, but the confidence intervals are much larger and noisy than for the other two approaches.

To further evaluate the three approaches, the performance indicators RMSE and $M A_{0.9}$ are also computed in correspondence of the four life fractions $\beta_{1}=0.3, \beta_{2}=0.6$, $\beta_{3}=0.8$ and $\beta_{4}=0.95$; since only 8 trajectories are available, to correctly verify the reliability of the method, the coverage indicator $\operatorname{Cov}_{0.9}$ is evaluated over all the predictions performed at each time instant $\tau_{j}$. Fig. 17 shows these results and compares the coverage indicator $\mathrm{Cov}_{0.9}$ with the target value $\alpha=0.9$, and the RMSE and $M A_{0.9}$ indicators with the average value $m R U L$ of the filters RUL at each life fraction $\beta$.
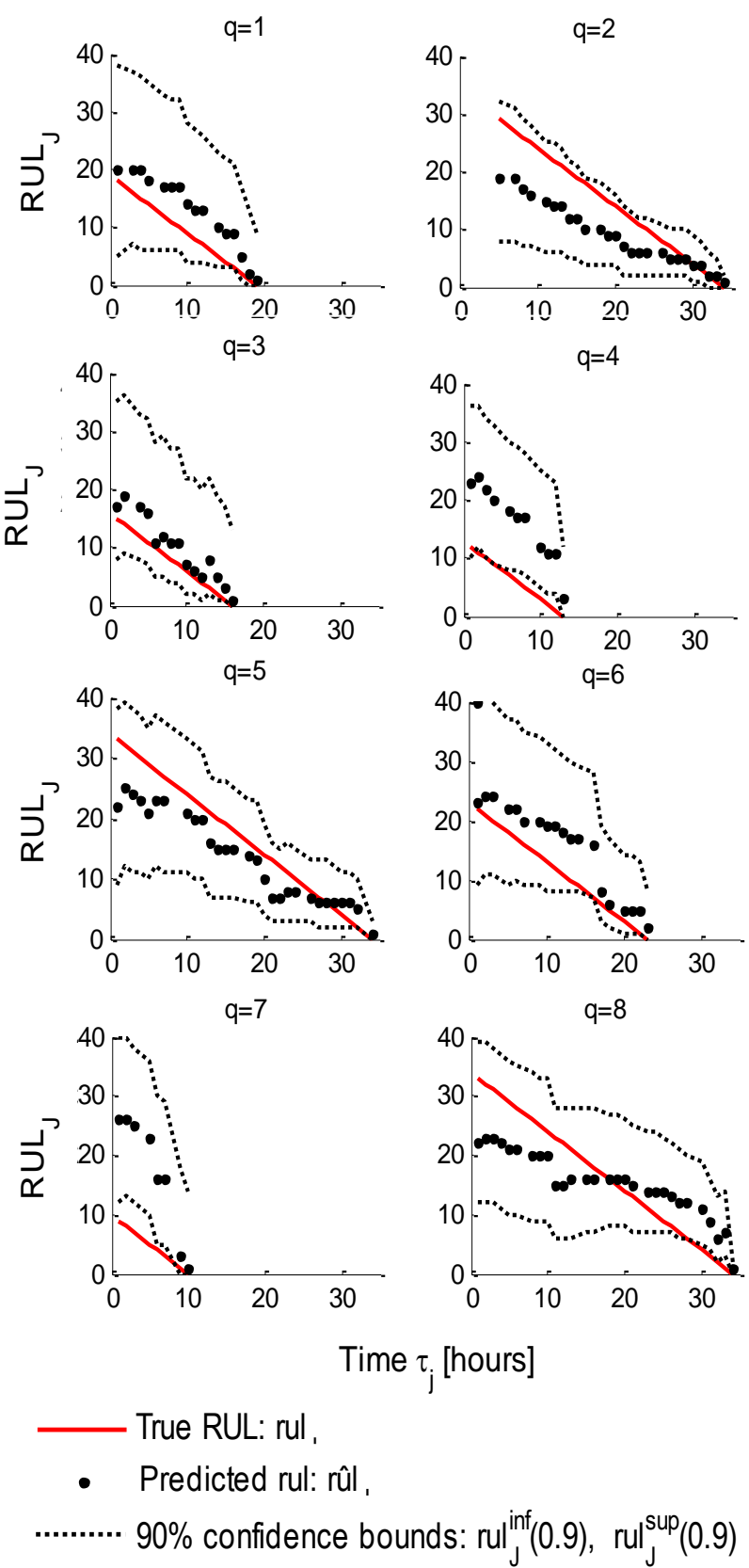

Fig. 14 Comparison of the RUL prediction supplied by approaches 1 (dots) and of its confidence bounds (dotted line) with the true RUL value.

Results show that the coverage is very close to the target value $\alpha=0.9$ for all three approaches. The prediction error (RMSE), however, is always quite large, ranging from being about half of the average RUL value $m R U L$ at the beginning of the clogging process $\left(\beta_{1}=0.3\right.$ ) up to almost three times 
(approach 1) this value at $\beta_{4}=0.95$. Approach 3 achieves always the highest accuracy, contrarily to approach 1 that obtains always the largest RMSE. On the other hand, approach 1 assures the narrowest confidence intervals for the value of the filters RUL, although their amplitude is still many times the average RUL value $m R U L$.

In the context of filter clogging, due to the large uncertainties involved, we believe that the capability of providing narrower confidence intervals (but still, reliable) of approach 1 should be preferred to the higher prediction accuracy of approaches 2 and 3 .
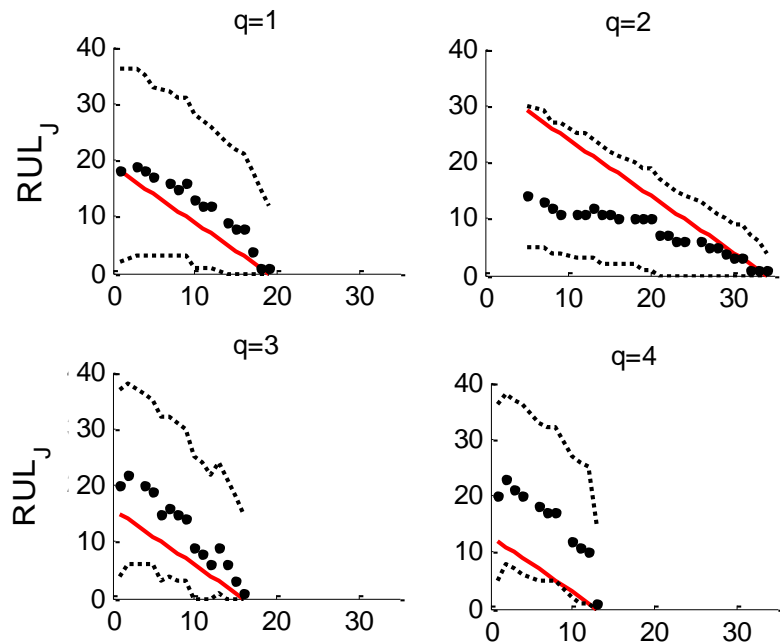

$q=4$
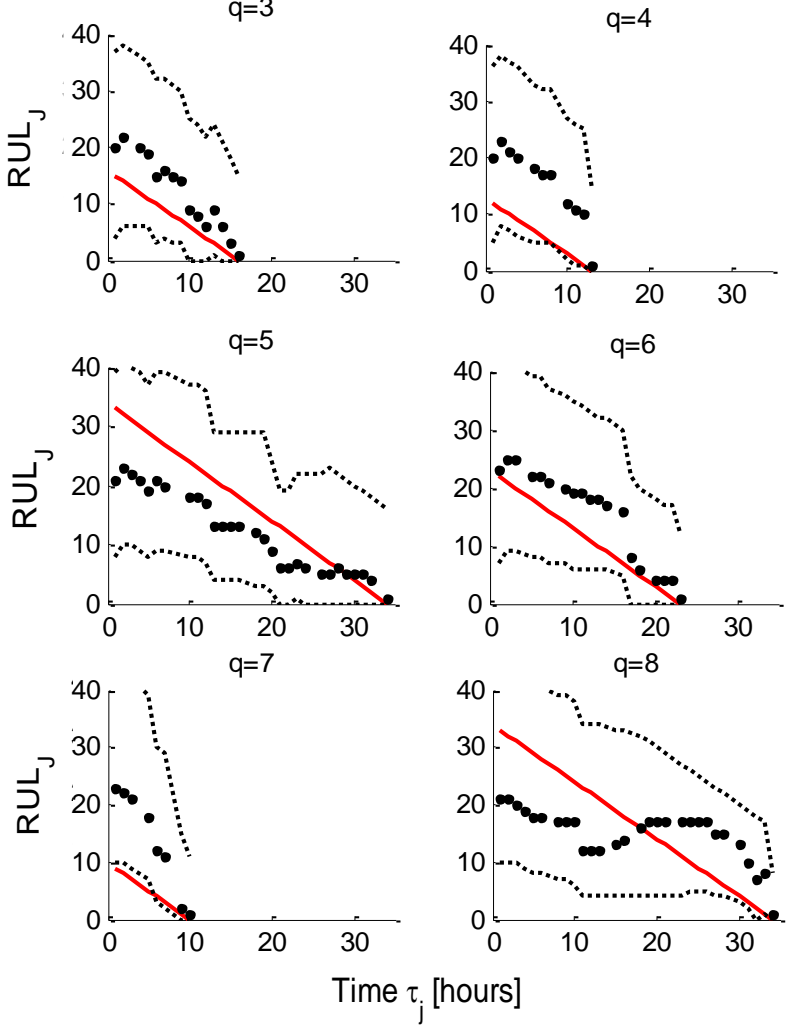

True RUL: rul

- Predicted rul: rûl,

........ 90\% confidence bounds: rul $_{j}^{\text {inf }}(0.9)$, rul $_{J}^{\text {sup }}(0.9)$

Fig. 15 Comparison of the RUL prediction supplied by approaches 2 (dots) and of its confidence bounds (dotted line) with the true RUL value.
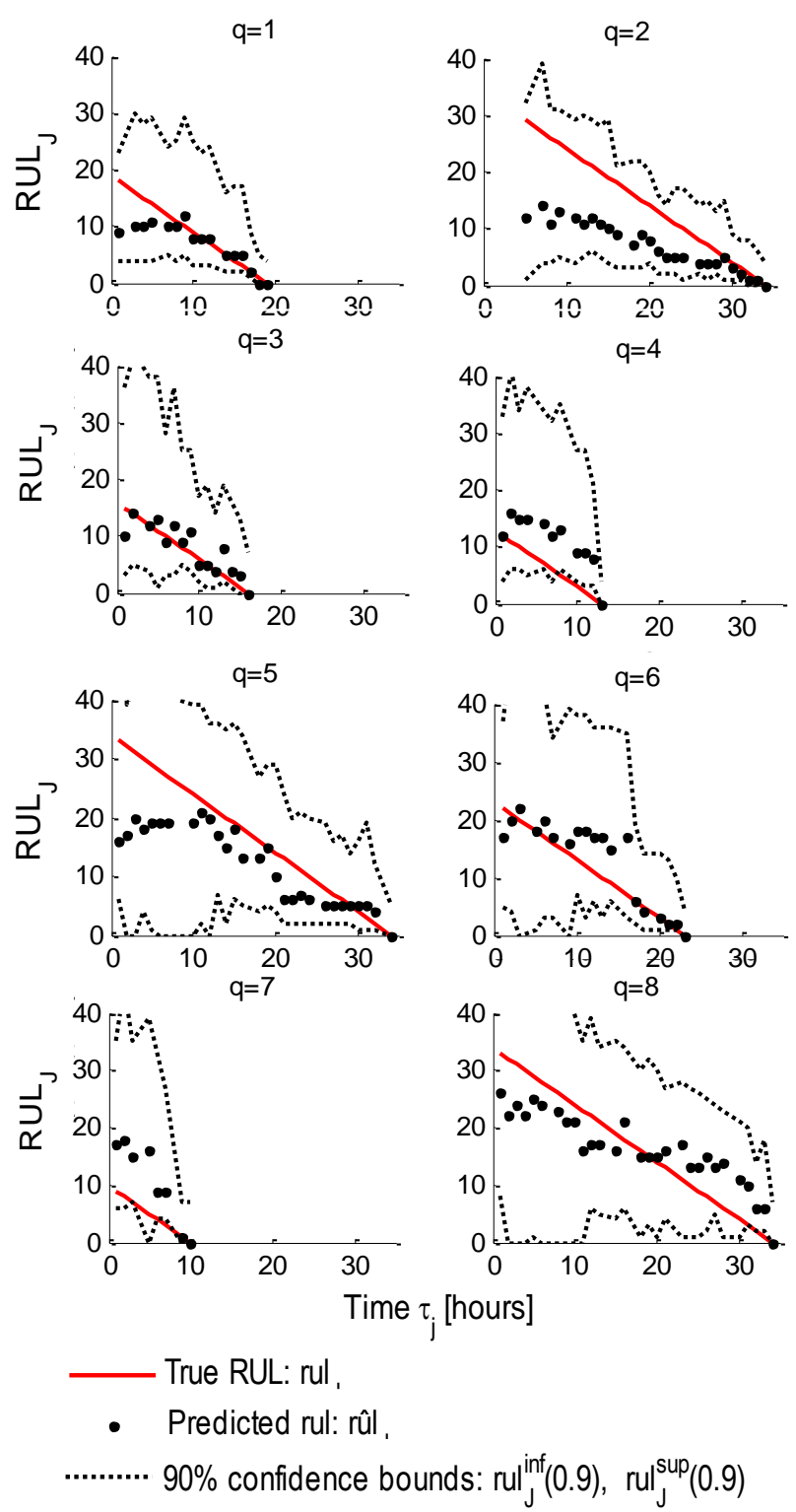

Fig. 16 Comparison of the RUL prediction supplied by approaches 3 (dots) and of its confidence bounds (dotted line) with the true RUL value.

\section{CONCLUSIONS}

In this work, we have considered the problem of directly predicting the RUL of degrading equipment and providing a measure of confidence in the prediction, based on a set of training degradation trajectories observed in similar equipments. We have resorted to Gaussian Process Regression to model the evolution of the equipment degradation. GPR treats degradation as a random variable, rather than as a deterministic quantity, and thus is able to provide predictions about the distribution of future degradation states. By comparing these predictions with a failure threshold, it has been possible to obtain the RUL prediction in the form of a pdf, from which the derivation of confidence intervals for the value of the equipment RUL is straightforward. Moreover, an effective structure of the coverage function has been proposed to allow accounting both for the common structure underlying 
all degradation trajectories and the variations specific to each trajectory.
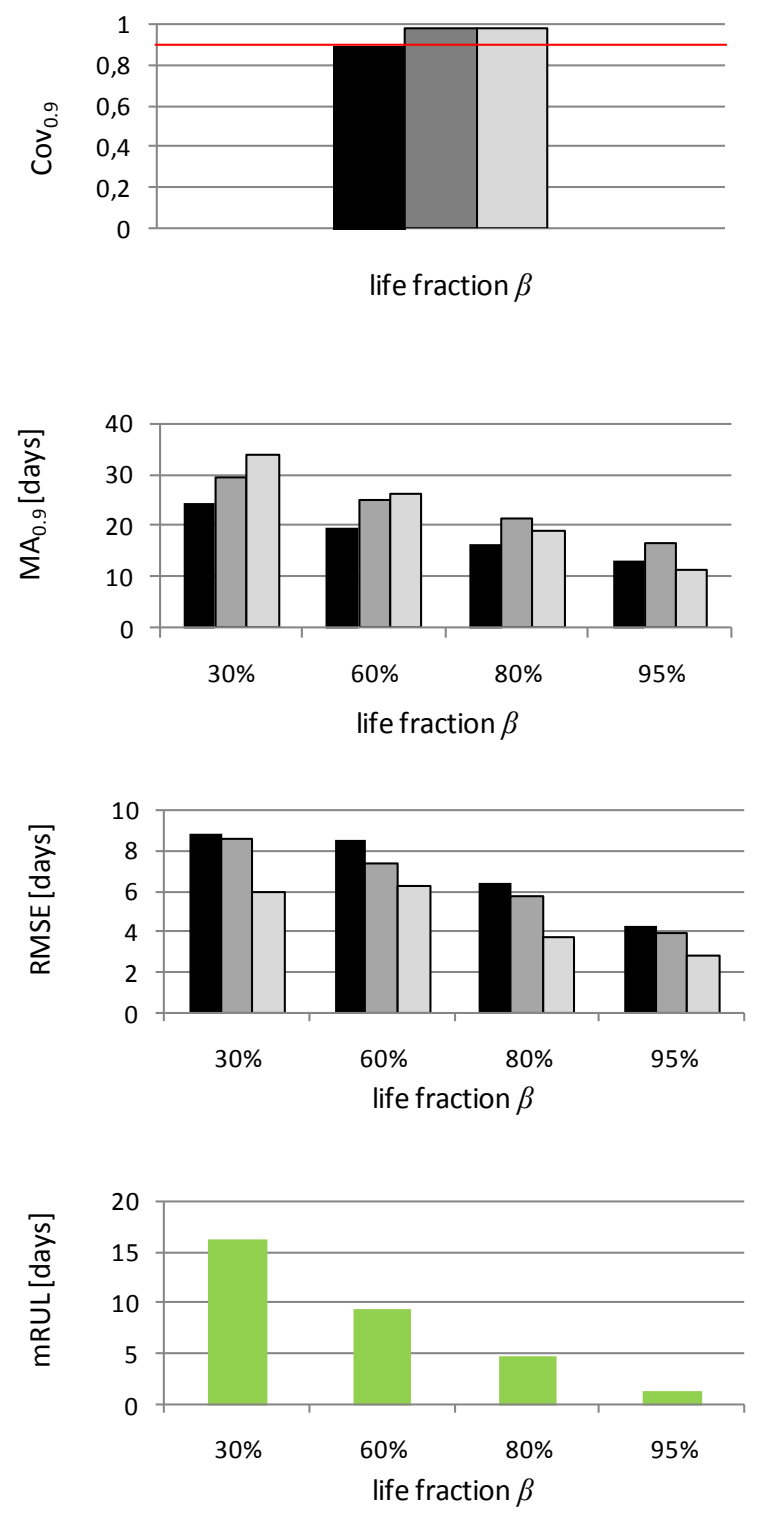

Approach $1 \quad \square$ Approach $2 \quad \square$ Approach $3 \square$ Average RUL

Fig. 17 Comparison of the performance of the three approaches on the $N^{\text {tst }}=8$ filter clogging trajectories. The horizontal line in the $\operatorname{Cov}_{0.9}$ figure (top, left) indicates the target coverage value $\alpha=0.9$.

Three different approaches to apply GPR to degradation modeling have been proposed and their performance compared in two case studies concerning the simulated accumulation of creep damage in ferritic steel and the real clogging of filters used to clean the sea water entering a BWR condenser, respectively. In both cases, the first approach proposed, which models the degradation state as a function of time, slightly outperforms the other two, which model the degradation rate as a function of time and as a function of the degradation state. However, also approaches 2 and 3 have shown the potentiality of providing accurate predictions and could prove to be more suited than approach 1 for treating other prognostic problems: for example, approach 3 could provide better results if the degradation processes would show a smaller noise and/or if the relation between degradation rate and degradation state were easier to model than the relation between degradation state and time.

In the application of GPR to prognostic problems characterized by high variability of the degradation trajectories, we could not always obtain the desired coverage of the predicted confidence interval. We interpreted this as an indication that some sources of uncertainty, e.g., the model uncertainty, have not been correctly accounted for. We suggest that resorting to the ensemble techniques [27,29], has the potential of improving these results.

The results obtained could also be affected by the choice of the covariance functions. Here, this choice has been done by a trial and error procedure, with the goal of maximizing the marginal likelihood of the GP with respect to the training data. The development of a procedure for the selection of GPR covariance functions is a fundamental requirement for the successful exploitation of GPR in prognostics.

A further important aspect of GPR is its computational cost, which scales typically as $O\left(N^{3}\right)$ with the number of training examples. In our application this was not a problem since we had, or chose, small training datasets, but it can make the application of the method prohibitive even on modern workstations for large problems (e.g. $N>10^{4}$ ). However, various methods have been suggested to reduce the problem by approximating the computations [22,29], and should be considered in future work to make GPR-based prognostics more efficient, especially if used in combination with Monte Carlo sampling (approach 3).

\section{APPENDIX A: MEAN AND COVARIANCE FUNCTIONS USED IN THE CASE STUDIES}

In the case studies considered in this work, combinations of the covariance functions listed in Table 1A have been used. Table $2 \mathrm{~A}$ and $3 \mathrm{~A}$ show the mean functions and the terms $k_{1}\left(x, x^{\prime}\right)$ and $k_{2}\left(x, x^{\prime}\right)$ of the covariance function in eq. (11) used in the case studies of Section III.A and Section III.B, respectively.

\section{APPENDIX B: DISTRIBUTION OF THE PARAMETERS IN THE CREEP GROWTH MODEL OF SECTION III.A.1}

All parameters $\varphi_{m 0}$ and $Q_{m}, m=1: 6$ which defines the quantities $\varphi_{1: 6}=A, B, H^{*}, h, K_{c}, C$ of the creep growth model in eq. (13), the temperature $T$ and the load $\sigma$ are assumed to have a Gaussian distribution with standard deviation equal to the $0.2 \%$ (low variability) or $0.5 \%$ (high variability) of their mean values given in Table $1 \mathrm{~B}$. 
TABLE IA

LIST OF THE BASIC COVARIANCE FUNCTIONS USED IN THE CASE STUDIES OF SECTION 3 ; SYMBOLS $b_{i}, i=1,2, \ldots$, ARE USED TO INDICATE THE HYPERPARAMETERS OF THE COVARIANCE FUNCTION

\begin{tabular}{lcc}
\hline \multicolumn{1}{c}{$\begin{array}{c}\text { Covariance } \\
\text { function }\end{array}$} & Symbol & Analytical expression of $k\left(x, x^{\prime}\right)$ \\
\hline \hline Constant & Const $\left(x, x^{\prime}\right)$ & $b_{1}$ \\
Linear & $\operatorname{Lin}\left(x, x^{\prime}\right)$ & $b_{1}\left(x \cdot x^{\prime}\right)$ \\
Polynomial & $\operatorname{Poly}_{d}\left(x, x^{\prime}\right)$ & $b_{1}\left(x \cdot x^{\prime}+b_{2}\right)^{d}$ \\
$\begin{array}{l}\text { Squared } \\
\text { exponential }\end{array}$ & $\operatorname{SE}\left(x, x^{\prime}\right)$ & $b_{1} \exp \left(\frac{-\left(x-x^{\prime}\right)^{2}}{b_{2}}\right)$ \\
$\begin{array}{l}\text { Matérn with } \\
v=\frac{3}{2}\end{array}$ & $\operatorname{Mat}_{3}\left(x, x^{\prime}\right)$ & $b_{1}\left(1+\frac{\sqrt{3}}{b_{2}}\left|x-x^{\prime}\right|\right) \exp \left(-\frac{\sqrt{3}}{b_{2}}\left|x-x^{\prime}\right|\right)$ \\
$\begin{array}{l}\text { Rational } \\
\text { quadratic }\end{array}$ & $\operatorname{RQ}\left(x, x^{\prime}\right)$ & $b_{1}\left(1+\frac{\left(x-x^{\prime}\right)^{2}}{2 b_{2} b_{3}}\right)^{-b_{2}}$ \\
& & $b_{1} \sin ^{-1}\left(\frac{b_{2} x x^{\prime}}{\sqrt{\left(1+b_{2} x^{2}\right)\left(1+b_{2} x^{\prime 2}\right)}}\right)$ \\
Neural Network & $\mathbf{N N}\left(x, x^{\prime}\right)$ & \\
\hline
\end{tabular}

TABLE IIA

LIST OF FUNCTIONS USED TO DEFINE THE PRIOR ON THE GP MEAN AND COVARIANCE IN THE CREEP GROWTH CASE STUDY (SECTION 3.1); SYMBOLS $a_{i}, i=1,2, \ldots$, ARE USED TO INDICATE THE HYPER-PARAMETERS OF THE MEAN FUNCTIONS

\begin{tabular}{cccc}
\hline Approach & $\begin{array}{c}\text { Mean function } \\
m(x)=\end{array}$ & \multicolumn{2}{c}{$\begin{array}{c}\text { Terms of the covariance } \\
\text { function in eq. (11) }\end{array}$} \\
\hline \hline & $k_{1}\left(x, x^{\prime}\right)=$ & $k_{2}\left(x, x^{\prime}\right)=$ \\
2 & $\sum_{i=0}^{3} a_{i} x^{i}$ & $\operatorname{Mat}_{3 / 2}\left(x, x^{\prime}\right)$ & $\mathrm{RQ}\left(x, x^{\prime}\right)+\operatorname{Poly}_{3}\left(x, x^{\prime}\right)$ \\
3 & $\sum_{i=0}^{2} a_{i} x^{i}$ & $\operatorname{Mat}_{3 / 2}\left(x, x^{\prime}\right)$ & $\mathrm{RQ}\left(x, x^{\prime}\right)+\operatorname{Poly}_{2}\left(x, x^{\prime}\right)$ \\
& $a_{1} x$ & $\mathbf{N N}\left(x, x^{\prime}\right)$ & $\operatorname{Poly}_{1}\left(x, x^{\prime}\right)$ \\
\hline
\end{tabular}

TABLE IIIA

LIST OF FUNCTIONS USED TO DEFINE THE PRIOR ON THE GP MEAN AND COVARIANCE IN THE CLOGGING FILTERS CASE STUDY (SECTION 3.2); SYMBOLS $a_{i}, i=1,2, \ldots$, ARE USED TO INDICATE THE HYPER-PARAMETERS OF

\begin{tabular}{cccc} 
& \multicolumn{3}{c}{ THE MEAN FUNCTIONS } \\
\hline Approach & $\begin{array}{c}\text { Mean function } \\
m(x)=\end{array}$ & \multicolumn{2}{c}{$\begin{array}{c}\text { Terms of the covariance } \\
\text { function in eq. (11) }\end{array}$} \\
& $k_{1}\left(x, x^{\prime}\right)=$ & $k_{2}\left(x, x^{\prime}\right)=$ \\
\hline \hline 1 & $\sum_{i=0}^{2} a_{i} x^{i}$ & $\mathrm{SE}\left(x, x^{\prime}\right)$ & $\mathbf{N N}\left(x, x^{\prime}\right)+\operatorname{Poly}_{1}\left(x, x^{\prime}\right)$ \\
& $a_{0}+a_{1} x$ & $\mathrm{SE}\left(x, x^{\prime}\right)$ & $\begin{array}{c}\text { Const }\left(x, x^{\prime}\right) \times \operatorname{Lin}\left(x, x^{\prime}\right) \\
\times \delta\left(x, x^{\prime}\right)\end{array}$ \\
3 & $a_{1} x$ & $\operatorname{Poly}_{1}\left(x, x^{\prime}\right)$ & $\operatorname{Poly}\left(x, x^{\prime}\right)$ \\
\hline
\end{tabular}

TABLE IB

MEAN VALUES OF PARAMETERS $\varphi_{m 0}$ AND $Q_{m}, m=1: 6$ OF THE CREEP GROWTH MODEL IN EQ. (13)

\begin{tabular}{cccc}
\hline Parameter & Mean value & Parameter & Mean value \\
\hline \hline$\varphi_{10}\left[\mathrm{~h}^{-1}\right]$ & $1.5 \times 10^{-5}$ & $\sigma_{[\mathrm{MPa}]}$ & 100 \\
$\varphi_{20}\left[\mathrm{MPa}^{-1}\right]$ & 50 & $Q_{1}$ & $1.9 \times 10^{4}$ \\
$\varphi_{30}$ & 5.6 & $Q_{2}$ & 3450 \\
$\varphi_{40}[\mathrm{MPa}]$ & $9.5 \times 10^{11}$ & $Q_{3}$ & 1450 \\
$\varphi_{50}\left[\mathrm{~h}^{-1}\right]$ & $3.2 \times 10^{6}$ & $Q_{4}$ & 9800 \\
$\varphi_{60}$ & $3 \times 10^{-5}$ & $Q_{5}$ & $1.45 \times 10^{4}$ \\
$T\left[{ }^{\circ} \mathrm{C}\right]$ & 565 & $Q_{6}$ & 6850 \\
\hline
\end{tabular}

\section{ACKNOWLEDGMENTS}

The work of Francesca Mangili has been supported by a $\mathrm{PhD}$ grant of the Institutt For Energiteknikk (IFE), OECD Halden Reactor Project, which also supplied the data for the filter clogging case study. The authors wish to thank Giulio Gola and Bent H. Nystad from IFE for their contribution to this work. The participation of Enrico Zio to this research is partially supported by the China NSFC under grant number 71231001.

\section{REFERENCES}

[1] Choi, S.S., Kang, K. S., Kim, H. G., Chang, S. H., 1995, Development of an on-line fuzzy expert system for integrated alarm processing in nuclear power plants, IEEE Transactions on Nuclear Science, Vol. 42 (4), pp. 1406-1418.

[2] Razavi-Far, R., Baraldi, P., Zio, E., 2012, Dynamic weighting ensembles for incremental learning and diagnosing new concept class faults in nuclear power systems, IEEE Transactions on Nuclear Science, Vol. 59 (5) pp. 2520-2530.

[3] Li, F., Upadhyaya, B.R., Perillo, S.R.P., 2012 Fault diagnosis of helical coil steam generator systems of an integral pressurized water reactor using optimal sensor selection, IEEE Transactions on Nuclear Science, 59 (2), pp. 403-410.

[4] Moshkbar-Bakhshayesh, K., Ghofrani, M.B., 2013, Transient identification in nuclear power plants: A review, Progress in Nuclear Energy, Vol. 67, pp. 23-32.

[5] Ma, J., Jiang, J., 2011, Applications of fault detection and diagnosis methods in nuclear power plants: A review, Progress in Nuclear Energy, 53 (3), pp. 255-266.

[6] Zio, E., Baraldi, P., 2005, Evolutionary fuzzy clustering for the Classification of transients in nuclear components, Progress in Nuclear Energy, Vol. 46 (3-4), pp. 282-296.

[7] Marseguerra, M., Zio, E., Baraldi, P., Oldrini, A. Fuzzy logic for signal prediction in nuclear systems (2003) Progress in Nuclear Energy, 43 (14 SPEC), pp. 373-380.

[8] Bond, L.J.; Ramuhalli, P.; Tawfik, M.S.; Lybeck, N.J.; , "Prognostics and life beyond 60 years for nuclear power plants," Prognostics and Health Management (PHM), 2011 IEEE Conference, pp.1-7, 20-23 June 2011

[9] Hines, J.W., Usynin, A., 2008. Current Computational Trends in Equipment Prognostics, Int. J. Comput. Intell. Syst., 1(1), 94-102.

[10] Zio, E., 2012 Prognostics and Health Management of Industrial Equipment. In: Kadry S, editor. Diagnostics and Prognostics of Engineering Systems: Methods and Techniques, IGI-Global. 
[11] Muller, A., Sunher, M., Iung, B., 2008. Formalization of a new prognosis model for supporting proactive maintenance implementation on industrial system. Reliability Engineering \& system Safety, Vol 93, pp. 234-253.

[12] Li, W., Pham, H., 2005. An inspection-maintenance model for systems with multiple competing processes. IEEE Transactions on Reliability, vol 54(2), pp. 318-27.

[13] Baraldi P., Mangili F., Zio E., 2013b. Investigation of uncertainty treatment capability of model-based and data-driven prognostic methods using simulated data. Reliability Engineering and System Safety, Vol 112C, pp. 94-108.

[14] Peel, L., 2008. Data Driven Prognostics using a Kalman Filter Ensemble of Neural Network Models, International Conference on Prognostics and Health Management, 06-09 oct, Denver, CO.

[15] Santosh, T.V., Srivastava, A., Sanyasi Rao, V.V.S., Gosh, A. K., Kushwaha, H.S., 2009. Diagnostic System for Identification of Accident Scenarios in Nuclear Power Plants using Artificial Neural Networks, Reliability Engineering and System Safety, 94, 759-762.

[16] Wang, T., Yu, J., Siegel, D., Lee, J., 2008. A Similarity-Based Prognostics Approach for Remaining Useful Life Estimation of Engineered Systems. International Conference on Prognostics and Health Management, 6-9 Oct., Denver (CO).

[17] Zio, E., Di Maio, F., 2010. A data-driven fuzzy approach for predicting the remaining useful life in dynamic failure scenarios of a nuclear system. Reliability Engineering \& System Safety, Vol. 95(1), pp. 49-57.

[18] Vachtsevanos, G., Wang, P., 2001. Fault prognosis using dynamic wavelet neural networks. Proceedings of IEEE Systems Readiness Technology Conference (AUTEST), pp 857-870.

[19] Goebel, K., Saha, B., Saxena, A., 2008. A Comparison of Three DataDriven Techniques for Prognostics, proc. of the 62nd Meeting of the Society For Machinery Failure Prevention Technology (MFPT), May 68, Virginia Beach, Virginia.

[20] Tipping, M.E., 2001. Sparse Bayesian Learning and the Relevance Vector Machine. Journal of Machine Learning Research, 1:211-244.

[21] MacKay, D.J.C., 1998. Introduction to Gaussian processes. In:Bishop CM (ed) Neural networks and machine learning, vol 168. NATO ASI Series, Springer, Berlin, pp 133-165

[22] Rasmussen, C., Williams, C., 2006. Gaussian processes for machine learning. MIT Press, Cambridge, MA.

[23] Liu, R., Ma, L., Kang, R., Wang, N., 2011. The modeling method on failure prognostics uncertainties in maintenance policy decision process. Proceedings of the 9th International Conference on Reliability, Maintenance and Safety (ICRMS), Jun 12-15; Guiyang, China.

[24] Tang, L., Kacprzynski, G.J., Goebel, K., Vachtsevanos, G., 2009. Methodologies for uncertainty management in prognostics. Proc IEEE Aerospace Conference, Mar 7-14; Big Sky, MT.

[25] Mohanty, S., Chattopadhyay, A., Peralta, P., Das, S., 2011. Bayesian Statistic Based Multivariate Gaussian Process Approach for Offline/Online Fatigue Crack Growth Prediction. Experimental Mechanics, Vol. 51, pp. 833-843.

[26] Miranda, E., Cester, A., Suñé, J., Paccagnella, A., Ghidini, G., 2005, Simulation of the time-dependent breakdown characteristics of heavyion irradiated gate oxides using a mean-reverting Poisson-Gaussian process, IEEE Transactions on Nuclear Science, Vol. 52 (5), pp. 14621467.

[27] Baraldi, P., Cadini, F., Mangili, F., Zio, E., 2013a. Model-Based and Data-Driven Prognostics under Different Available Information. accepted for publication in Probabilistic Engineering Mechanics.

[28] Mann, R., Freeman, R., Osborne, M., Garnett, R., Armstrong, C., Meade, J., Biro, D., Guilford, T., Roberts, S., 2011. Objectively identifying landmark use and predicting flight trajectories of the homing pigeon using Gaussian processes, Journal of the Royal Society Interface, Vol. 8, pp. 210-219.

[29] Shi, J.Q., Murray-Smith, R., Titterington, D.M., 2005. Hierarchical Gaussian process mixtures for regression, Statistics and Computing, Vol. 15 , pp. 31-41.

[30] Mustata, R., Hayhurst, D.R., 2005. Creep constitutive equations for a $0.5 \mathrm{Cr} 0.5 \mathrm{Mo} 0.25 \mathrm{~V}$ ferritic steel in the temperature range $5658 \mathrm{C}-675$ 8C. International Journal of Pressure Vessels and Piping, Vol 82, pp. 363-372.

[31] Song, C.B., Park, H.S., Lee, K.W., 2006. Experimental study of clogging with monodisperse PSL particles. Power Technology, Vol. 163, pp. 152-159.

[32] Contal, P., Simao, J., Thomas, D., Frising, T., Callé, S., Appert-Collin, J.C., Bémer, D., 2004. Clogging of fibre filters by submicron droplets.
Phenomena and influence of operating conditions. Aerosol Science, Vol. 35, pp. 263-278.

[33] Nystad, B.H., 2009. Condition-Based Maintenance (CBM) - filter clogging at OKG 1, a case study, HWR-961, OECD Halden Reactor Project. 